ESAIM: M2AN 55 (2021) 57-76

https://doi.org/10.1051/m2an/2020075
ESAIM: Mathematical Modelling and Numerical Analysis

www.esaim-m2an.org

\title{
ELECTROMAGNETIC STEKLOV EIGENVALUES: APPROXIMATION ANALYSIS
}

\author{
MARTIN HALlA ${ }^{1,2, *}$
}

\begin{abstract}
We continue the work of Camano et al. [SIAM J. Math. Anal. 49 (2017) 4376-4401] on electromagnetic Steklov eigenvalues. The authors recognized that in general the eigenvalues do not correspond to the spectrum of a compact operator and hence proposed a modified eigenvalue problem with the desired properties. The present article considers the original and the modified electromagnetic Steklov eigenvalue problem. We cast the problems as eigenvalue problem for a holomorphic operator function $A(\cdot)$. We construct a "test function operator function" $T(\cdot)$ so that $A(\lambda)$ is weakly $T(\lambda)$-coercive for all suitable $\lambda$, i.e. $T(\lambda)^{*} A(\lambda)$ is a compact perturbation of a coercive operator. The construction of $T(\cdot)$ relies on a suitable decomposition of the function space into subspaces and an apt sign change on each subspace. For the approximation analysis, we apply the framework of T-compatible Galerkin approximations. For the modified problem, we prove that convenient commuting projection operators imply T-compatibility and hence convergence. For the original problem, we require the projection operators to satisfy an additional commutator property which concerns the tangential trace. The existence and construction of such projection operators remain open questions.
\end{abstract}

Mathematics Subject Classification. 35J25, 35R30, 65H17, 65N25.

Received October 7, 2019. Accepted November 10, 2020.

\section{INTRODUCTION}

Novel nondestructive evaluation methods based on inverse scattering [9] give rise to a multitude of new eigenvalue problems. Among these are so-called transmission eigenvalue problems [11] and Steklov eigenvalue problems [10]. Not all of these eigenvalue problems fall into classes which are covered in classical literature. Among the important questions on these eigenvalue problems are

- Fredholmness,

- discreteness of the spectrum,

- existence of eigenvalues,

- estimates on the number of eigenvalues,

- properties of the eigenvalue distribution, such as accumulation points and eigenvalue free zones,

- results on the completeness of eigenfunctions,

- and reliable computational approximations.

Keywords and phrases. Steklov eigenvalues, nondestructive testing, T-coercivity.

1 Max-Planck-Institut für Sonnensystemforschung, Justus-von-Liebig-Weg 3, 37077 Göttingen, Deutschland.

2 Institut für Numerik und Angewandte Mathematik, Geog-August-Universität Göttingen, Lotzestraße 16-18, 37083

Göttingen, Deutschland.

*Corresponding author: halla@mps.mpg.de 
The electromagnetic Steklov eigenvalue problem to find $(\lambda, u)$ so that

$$
\begin{aligned}
\operatorname{curl} \operatorname{curl} u-\omega^{2} \epsilon u=0 & \text { in } \Omega, \\
\nu \times \operatorname{curl} u+\lambda \nu \times u \times \nu=0 & \text { at } \partial \Omega .
\end{aligned}
$$

was considered in the recent publication [12]. Therein the authors of [12] considered the case that $\Omega$ is a ball and the material parameter $\epsilon$ is constant. For this setting they proved the existence of two infinite sequences of eigenvalues, one converging to zero and one converging to infinity. Consequently the eigenvalue problem can't be transformed to an eigenvalue problem for a compact operator. This observation led the authors of [12] to discard the original eigenvalue problem and to modify instead the boundary condition to

$$
\nu \times \operatorname{curl} u+\lambda S(\nu \times u \times \nu)=0 \quad \text { at } \partial \Omega .
$$

with a suitable operator $S$. The authors of [12] proved that the modified eigenvalue problem can indeed be transformed to an eigenvalue problem for a compact operator.

In this note we consider the original as well as the modified electromagnetic Steklov eigenvalue problem. We formulate the problems as holomorphic operator function eigenvalue problems to find $(\lambda, u) \in \mathbb{C} \times X$ so that $A(\lambda) u=0$.

We assume reasonable conditions on the material parameters and the domain to analyze the Fredholmness of $A(\lambda)$. We prove that for the original problem $A(\lambda)$ is Fredholm if and only if $\lambda \in \mathbb{C} \backslash\{0\}$, while for the modified problem $A(\lambda)$ is Fredholm for all $\lambda \in \mathbb{C}$. For our analysis we construct an operator function $T(\cdot)$ which is bijective at each $\lambda \in \mathbb{C} \backslash\{0\}$ (respective $\lambda \in \mathbb{C}$ ) so that $T(\lambda)^{*} A(\lambda)$ is a compact perturbation of a coercive operator. The construction of $T(\cdot)$ relies on a decomposition of the function space into subspaces and an apt sign change on each subspace.

We apply the framework of [17] to analyze the convergence of Galerkin approximations. To this end, we need to prove the existence of apt approximations of $T(\cdot)$. We prove for the modified problem, that the existence of convenient commuting projections imply the existence of such apt approximations of $T(\cdot)$. This implies the convergence of convenient $H$ (curl)-finite element methods. For the original problem, we require the projection operators to satisfy an additional commuting property, which concerns the tangential trace, to establish the same result. Thus it is unclear if common $H$ (curl)-finite element methods converge. Further numerical studies are necessary to determine if this is the case or not. If common $H$ (curl)-finite element methods fail, then our analysis may raise ideas for the construction of new suitable elements. On the other hand, if common $H$ (curl)finite element methods work, then it is likely that such commuting projection operators exist. If the existence of such commuting projection operators can be proven, then this would complete our presented convergence analysis.

We report on the existence of eigenvalues and properties of their distribution of the electromagnetic Steklov eigenvalue problems in the self adjoint case in the companion article [16]. In particular, we report therein that the spectrum of the original problem consists of three disjoint parts: The essential spectrum consisting of the point zero, an infinite sequence of positive eigenvalues which accumulate only at infinity and an infinite sequence of negative eigenvalues which accumulate only at zero. The spectrum of the modified problem consists of an infinite sequence of eigenvalues which accumulate only at positive infinity.

The remainder of this article is organized as follows. In Section 2 we set our notation and formulate our assumptions on the domain and the material parameters. We also recall some classic regularity, embedding and decomposition results which will be essential for our analysis. In Section 3 we introduce the considered electromagnetic Steklov eigenvalue problem and define the associated holomorphic operator function $A_{X}(\cdot)$. We define $T(\cdot)$ and prove that $A_{X}(\cdot)$ is weakly $T(\cdot)$-coercive on $\mathbb{C} \backslash\{0\}$ while $A_{X}(0)$ is not Fredholm. In Section 4 we prove that Galerkin approximations which admit uniformly bounded commuting projections are asymptotically (with respect to the discretization index) reliable. In Section 5 we introduce the modified electromagnetic Steklov eigenvalue problem and define the associated holomorphic operator function $\tilde{A}_{\tilde{X}}(\cdot)$. We define $\tilde{T}$ and prove that $\tilde{A}_{\tilde{X}}(\cdot)$ is weakly $\tilde{T}$-coercive. We introduce a reformulation of the eigenvalue problem by means of an 
operator function $\tilde{A}^{l}(\cdot)$, which avoids the explicit appearance of $S$. Likewise we define $\tilde{T}^{l}(\cdot)$ and prove that $\tilde{A}^{l}(\cdot)$ is weakly $\tilde{T}^{l}(\cdot)$-coercive. In Section 6 we prove that Galerkin approximations which admit uniformly bounded commuting projections are asymptotically (with respect to the discretization index) reliable. We further discuss the computational implementation of the Galerkin approximations. We conclude in Section 7.

\section{General Setting}

In this section we set our notation, formulate assumptions on the domain and material parameters and recall necessary results from different literature.

\subsection{Functional analysis}

For generic Banach spaces $\left(X,\|\cdot\|_{X}\right),\left(Y,\|\cdot\|_{Y}\right)$ denote $L(X, Y)$ the space of all bounded linear operators from $X$ to $Y$ with operator norm $\|A\|_{L(X, Y)}:=\sup _{u \in X \backslash\{0\}}\|A u\|_{Y} /\|u\|_{X}, A \in L(X, Y)$. We further set $L(X):=$ $L(X, X)$. For generic Hilbert spaces $\left(X,\langle\cdot, \cdot\rangle_{X}\right),\left(Y,\langle\cdot, \cdot\rangle_{Y}\right)$ and $A \in L(X, Y)$ we denote $A^{*} \in L(Y, X)$ its adjoint operator defined through $\left\langle u, A^{*} u^{\prime}\right\rangle_{X}=\left\langle A u, u^{\prime}\right\rangle_{Y}$ for all $u \in X, u^{\prime} \in Y$. We say that an operator $A \in L(X)$ is coercive if $\inf _{u \in X \backslash\{0\}}\left|\langle A u, u\rangle_{X}\right| /\|u\|_{X}^{2}>0$. We say that $A \in L(X)$ is weakly coercive, if there exists a compact operator $K \in L(X)$ so that $A+K$ is coercive. For bijective $T \in L(X)$ we say that $A$ is (weakly) $T$-coercive, if $T^{*} A$ is (weakly) coercive. Let $\Lambda \subset \mathbb{C}$ be open and connected and consider operator functions $A(\cdot), T(\cdot): \Lambda \rightarrow L(X)$ so that $T(\lambda)$ is bijective for all $\lambda \in \Lambda$. We call $A(\cdot)$ (weakly) $(T(\cdot)$-)coercive if $A(\lambda)$ is (weakly) (T( $\lambda)$-)coercive for all $\lambda \in \Lambda$. We denote the spectrum of $A(\cdot)$ as $\sigma(A(\cdot)):=\{\lambda \in \Lambda: A(\lambda)$ is not bijective $\}$ and the resolvent set as $\rho(A(\cdot)):=\Lambda \backslash \sigma(A(\cdot))$. For a closed subspace $X_{n} \subset X$ denote $P_{n} \in L\left(X, X_{n}\right)$ the orthogonal projection. Consider $A \in L(X)$ to be weakly $T$-coercive. For a sequence $\left(X_{n}\right)_{n \in \mathbb{N}}$ of finite dimensional subspaces $X_{n} \subset X$ with $\lim _{n \in \mathbb{N}}\left\|u-P_{n} u\right\|_{X}=0$ for each $u \in X$, we say that the Galerkin approximation $\left.P_{n} A\right|_{X_{n}} \in L\left(X_{n}\right)$ is $T$-compatible, if there exists a sequence $\left(T_{n}\right)_{n \in \mathbb{N}}, T_{n} \in L\left(X_{n}\right)$ so that

$$
\left\|T-T_{n}\right\|_{n}:=\sup _{u_{n} \in X_{n} \backslash\{0\}}\left\|\left(T-T_{n}\right) u_{n}\right\|_{X} /\left\|u_{n}\right\|_{X}
$$

tends to zero as $n \rightarrow \infty$. Let $A(\cdot): \Lambda \rightarrow L(X)$ be weakly $T(\cdot)$-coercive. We say that the Galerkin approximation $\left.P_{n} A(\cdot)\right|_{X_{n}}: \Lambda \rightarrow L\left(X_{n}\right)$ is $T(\cdot)$-compatible, if $\left.P_{n} A(\lambda)\right|_{X_{n}} \in L\left(X_{n}\right)$ is $T(\lambda)$-compatible for each $\lambda \in \Lambda$.

We recall from Corollary 2.8 of [17]: Let $A(\cdot): \Lambda \rightarrow L(X)$ be a weakly $T(\cdot)$-coercive holomorphic operator function with non-empty resolvent set and $A_{n}(\cdot): \Lambda \rightarrow L\left(X_{n}\right)$ be a $T(\cdot)$-compatible Galerkin approximation. Then

(i) For every eigenvalue $\lambda_{0}$ of $A(\cdot)$ exists a sequence $\left(\lambda_{n}\right)_{n \in \mathbb{N}}$ converging to $\lambda_{0}$ with $\lambda_{n}$ being an eigenvalue of $A_{n}(\cdot)$ for almost all $n \in \mathbb{N}$.

(ii) Let $\left(\lambda_{n}, u_{n}\right)_{n \in \mathbb{N}}$ be a sequence of normalized eigenpairs of $A_{n}(\cdot)$, i.e.

$$
A_{n}\left(\lambda_{n}\right) u_{n}=0
$$

and $\left\|u_{n}\right\|_{X}=1$, so that $\lambda_{n} \rightarrow \lambda_{0} \in \Lambda$, then

(a) $\lambda_{0}$ is an eigenvalue of $A(\cdot)$,

(b) $\left(u_{n}\right)_{n \in \mathbb{N}}$ is a compact sequence and its cluster points are normalized eigenelements of $A\left(\lambda_{0}\right)$.

(iii) For every compact $\tilde{\Lambda} \subset \rho(A)$ the sequence $\left(A_{n}(\cdot)\right)_{n \in \mathbb{N}}$ is stable on $\tilde{\Lambda}$, i.e. there exist $n_{0} \in \mathbb{N}$ and $c>0$ such that $\left\|A_{n}(\lambda)^{-1}\right\|_{L\left(X_{n}\right)} \leq c$ for all $n>n_{0}$ and all $\lambda \in \tilde{\Lambda}$.

(iv) For every compact $\tilde{\Lambda} \subset \Lambda$ with rectifiable boundary $\partial \tilde{\Lambda} \subset \rho(A(\cdot))$ such that $\sigma(A(\cdot)) \cap \tilde{\Lambda}=\left\{\lambda_{0}\right\}$ exists an index $n_{0} \in \mathbb{N}$ such that

$$
\operatorname{dim} G\left(A(\cdot), \lambda_{0}\right)=\sum_{\lambda_{n} \in \sigma\left(A_{n}(\cdot)\right) \cap \tilde{\Lambda}} \operatorname{dim} G\left(A_{n}(\cdot), \lambda_{n}\right)
$$


for all $n>n_{0}$, whereby $G(B(\cdot), \lambda)$ denotes the generalized eigenspace of an operator function $B(\cdot)$ at $\lambda \in \Lambda$.

Let $\tilde{\Lambda} \subset \Lambda$ be a compact set with rectifiable boundary $\partial \tilde{\Lambda} \subset \rho(A(\cdot)), \tilde{\Lambda} \cap \sigma(A(\cdot))=\left\{\lambda_{0}\right\}$ and

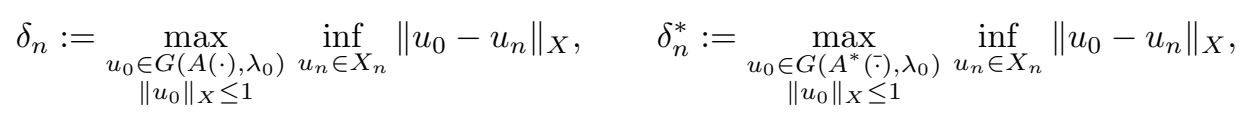

whereby $\overline{\lambda_{0}}$ denotes the complex conjugate of $\lambda_{0}$ and $A^{*}(\cdot)$ the adjoint operator function of $A(\cdot)$ defined by $A^{*}(\lambda):=A(\lambda)^{*}$ for each $\lambda \in \Lambda$. Then there exist $n \in \mathbb{N}$ and $c>0$ such that for all $n>n_{0}$,

(v)

$$
\left|\lambda_{0}-\lambda_{n}\right| \leq c\left(\delta_{n} \delta_{n}^{*}\right)^{1 / \varkappa\left(A(\cdot), \lambda_{0}\right)}
$$

for all $\lambda_{n} \in \sigma\left(A_{n}(\cdot)\right) \cap \tilde{\Lambda}$, whereby $\varkappa\left(A(\cdot), \lambda_{0}\right)$ denotes the maximal length of a Jordan chain of $A(\cdot)$ at the eigenvalue $\lambda_{0}$,

$$
\left|\lambda_{0}-\lambda_{n}^{\text {mean }}\right| \leq c \delta_{n} \delta_{n}^{*}
$$

whereby $\lambda_{n}^{\text {mean }}$ is the weighted mean of all the eigenvalues of $A_{n}(\cdot)$ in $\tilde{\Lambda}$

$$
\lambda_{n}^{\text {mean }}:=\sum_{\lambda \in \sigma\left(A_{n}(\cdot)\right) \cap \tilde{\Lambda}} \lambda \frac{\operatorname{dim} G\left(A_{n}(\cdot), \lambda\right)}{\operatorname{dim} G\left(A(\cdot), \lambda_{0}\right)}
$$

$$
\inf _{u_{0} \in \operatorname{ker} A\left(\lambda_{0}\right)}\left\|u_{n}-u_{0}\right\|_{X} \leq c\left(\left|\lambda_{n}-\lambda_{0}\right|+\max _{\substack{u_{0}^{\prime} \in \operatorname{ker} A\left(\lambda_{0}\right) \\\left\|u_{0}^{\prime}\right\|_{X} \leq 1}} \inf _{u_{n}^{\prime} \in X_{n}}\left\|u_{0}^{\prime}-u_{n}^{\prime}\right\|_{X}\right) \leq c\left(c\left(\delta_{n} \delta_{n}^{*}\right)^{1 / \varkappa\left(A(\cdot), \lambda_{0}\right)}+\delta_{n}\right)
$$

for all $\lambda_{n} \in \sigma\left(A_{n}(\cdot)\right) \cap \tilde{\Lambda}$ and all $u_{n} \in \operatorname{ker} A_{n}\left(\lambda_{n}\right)$ with $\left\|u_{n}\right\|_{X}=1$.

\subsection{Lebesgue and Sobolev spaces}

Let $\Omega \subset \mathbb{R}^{3}$ be a bounded and path-connected Lipschitz domain and $\nu$ the outer unit normal vector at $\partial \Omega$. We use standard notation for Lebesgue and Sobolev spaces $L^{2}(\Omega), L^{\infty}(\Omega), W^{1, \infty}(\Omega), H^{s}(\Omega)$ defined on the domain $\Omega$ and $L^{2}(\partial \Omega), H^{s}(\partial \Omega)$ defined on the boundary $\partial \Omega$. We recall the continuity of the trace operator $\operatorname{tr} \in L\left(H^{s}(\Omega), H^{s-1 / 2}(\partial \Omega)\right)$ for all $s>1 / 2$. For a vector space $X$ of scalar valued functions we denote its bold symbol as space of three-vector valued functions $\mathbf{X}:=X^{3}=X \times X \times X$, e.g. $\mathbf{L}^{2}(\Omega), \mathbf{H}^{s}(\Omega), \mathbf{L}^{2}(\partial \Omega), \mathbf{H}^{s}(\partial \Omega)$. For $\mathbf{L}^{2}(\partial \Omega)$ or a subspace, e.g. $\mathbf{H}^{s}(\partial \Omega), s>0$, the subscript $t$ denotes the subspace of tangential fields. In particular $\mathbf{L}_{t}^{2}(\partial \Omega)=\left\{u \in \mathbf{L}^{2}(\partial \Omega): \nu \cdot u=0\right\}$ and $\mathbf{H}_{t}^{s}(\partial \Omega)=\left\{u \in \mathbf{H}^{s}(\partial \Omega): \nu \cdot u=0\right\}$. Let further $H_{0}^{1}(\Omega)$ be the subspace of $H^{1}(\Omega)$ of all functions with vanishing Dirichlet trace, $H_{*}^{1}(\Omega)$ be the subspace of $H^{1}(\Omega)$ of all functions with vanishing mean, i.e. $\langle u, 1\rangle_{L^{2}(\Omega)}=0$ and $H_{*}^{1}(\partial \Omega)$ be the subspace of $H^{1}(\partial \Omega)$ of all functions with vanishing mean $\langle u, 1\rangle_{L^{2}(\partial \Omega)}=0$.

\subsection{Additional function spaces}

Denote $\partial_{x_{i}} u$ the partial derivative of a function $u$ with respect to the variable $x_{i}$. Let

$$
\begin{aligned}
& \nabla u:=\left(\partial_{x_{1}} u, \partial_{x_{2}} u, \partial_{x_{3}} u\right)^{\top} \\
& \operatorname{div}\left(u_{1}, u_{2}, u_{3}\right)^{\top}:=\partial_{x_{1}} u_{1}+\partial_{x_{2}} u_{2}+\partial_{x_{3}} u_{3}, \\
& \operatorname{curl}\left(u_{1}, u_{2}, u_{3}\right)^{\top}:=\left(\partial_{x_{2}} u_{3}-\partial_{x_{3}} u_{2}, \partial_{x_{3}} u_{1}-\partial_{x_{1}} u_{3}, \partial_{x_{1}} u_{2}-\partial_{x_{2}} u_{1}\right)^{\top} .
\end{aligned}
$$


For a bounded Lipschitz domain $\Omega$ let $\nabla_{\partial}$, $\operatorname{div}_{\partial}$ and $\operatorname{curl}_{\partial}=\nu \times \nabla_{\partial}$ be the respective differential operators for functions defined on $\partial \Omega$. We recall that for $u \in \mathbf{L}^{2}(\Omega)$ with curl $u \in \mathbf{L}^{2}(\Omega)$ the tangential trace $\operatorname{tr}_{\nu \times} u \in \mathbf{H}^{-1 / 2}\left(\operatorname{div}_{\partial} ; \partial \Omega\right):=\left\{u \in \mathbf{H}^{-1 / 2}(\partial \Omega): \operatorname{div}_{\partial} u \in H^{-1 / 2}(\partial \Omega)\right\},\|u\|_{\mathbf{H}^{-1 / 2}\left(\operatorname{div}_{\partial} ; \partial \Omega\right)}^{2}:=\|u\|_{\mathbf{H}^{-1 / 2}(\partial \Omega)}^{2}+$ $\left\|\operatorname{div}_{\partial} u\right\|_{H^{-1 / 2}(\partial \Omega)}^{2}$ is well defined and $\left\|\operatorname{tr}_{\nu \times} u\right\|_{\mathbf{H}^{-1 / 2}\left(\operatorname{div}_{\partial} ; \partial \Omega\right)}^{2}$ is bounded by a constant times $\|u\|_{\mathbf{L}^{2}(\Omega)}^{2}+$ $\|\operatorname{curl} u\|_{\mathbf{L}^{2}(\Omega)}^{2}$. Likewise for $u \in \mathbf{L}^{2}(\Omega)$ with $\operatorname{div} u \in L^{2}(\Omega)$ the normal $\operatorname{trace} \operatorname{tr}_{\nu}$. $u \in H^{-1 / 2}(\partial \Omega)$ is well defined and $\left\|\operatorname{tr}_{\nu \cdot} u\right\|_{H^{-1 / 2}(\partial \Omega)}^{2}$ is bounded by a constant times $\|u\|_{\mathbf{L}^{2}(\Omega)}^{2}+\|\operatorname{div} u\|_{L^{2}(\Omega)}^{2}$. For $\mathrm{d} \in\left\{\right.$ curl, $\operatorname{div}, \operatorname{tr}_{\nu \times}, \operatorname{tr}_{\nu}$. $\}$ let

$$
L^{2}(\mathrm{~d}):=\left\{\begin{array}{l}
\mathbf{L}^{2}(\Omega), \quad \mathrm{d}=\operatorname{curl}, \\
L^{2}(\Omega), \mathrm{d}=\operatorname{div}, \\
\mathbf{L}_{t}^{2}(\partial \Omega), \mathrm{d}=\operatorname{tr}_{\nu \times}, \\
L^{2}(\partial \Omega), \mathrm{d}=\operatorname{tr}_{\nu} .
\end{array}\right.
$$

Let

$$
\begin{aligned}
H(\mathrm{~d} ; \Omega) & :=\left\{u \in L^{2}(\Omega): \mathrm{d} u \in L^{2}(\mathrm{~d})\right\}, \\
\left\langle u, u^{\prime}\right\rangle_{H(\mathrm{~d} ; \Omega)} & :=\left\langle u, u^{\prime}\right\rangle_{L^{2}(\Omega)}+\left\langle\mathrm{d} u, \mathrm{~d} u^{\prime}\right\rangle_{L^{2}(\mathrm{~d})}, \\
H\left(\mathrm{~d}^{0} ; \Omega\right) & :=\{u \in H(\mathrm{~d} ; \Omega): \mathrm{d} u=0\} .
\end{aligned}
$$

Also for

$$
\mathrm{d}_{1}, \mathrm{~d}_{2}, \mathrm{~d}_{3}, \mathrm{~d}_{4} \in\left\{\operatorname{curl}, \operatorname{div}, \operatorname{tr}_{\nu \times}, \operatorname{tr}_{\nu}, \operatorname{curl}^{0}, \operatorname{div}^{0}, \operatorname{tr}_{\nu \times}^{0}, \operatorname{tr}_{\nu}^{0}\right\}
$$

let

$$
\begin{aligned}
H\left(\mathrm{~d}_{1}, \mathrm{~d}_{2} ; \Omega\right):= & H\left(\mathrm{~d}_{1} ; \Omega\right) \cap H\left(\mathrm{~d}_{2} ; \Omega\right) \\
\left\langle u, u^{\prime}\right\rangle_{H\left(\mathrm{~d}_{1}, \mathrm{~d}_{2} ; \Omega\right)}:= & \left\langle u, u^{\prime}\right\rangle_{L^{2}(\Omega)}+\left\langle\mathrm{d}_{1} u, \mathrm{~d}_{1} u^{\prime}\right\rangle_{L^{2}\left(\mathrm{~d}_{1}\right)}+\left\langle\mathrm{d}_{2} u, \mathrm{~d}_{2} u^{\prime}\right\rangle_{L^{2}\left(\mathrm{~d}_{2}\right)}, \\
H\left(\mathrm{~d}_{1}, \mathrm{~d}_{2}, \mathrm{~d}_{3} ; \Omega\right):= & H\left(\mathrm{~d}_{1} ; \Omega\right) \cap H\left(\mathrm{~d}_{2} ; \Omega\right) \cap H\left(\mathrm{~d}_{3} ; \Omega\right) \\
\left\langle u, u^{\prime}\right\rangle_{H\left(\mathrm{~d}_{1}, \mathrm{~d}_{2}, \mathrm{~d}_{3} ; \Omega\right)}:= & \left\langle u, u^{\prime}\right\rangle_{L^{2}(\Omega)}+\left\langle\mathrm{d}_{1} u, \mathrm{~d}_{1} u^{\prime}\right\rangle_{L^{2}\left(\mathrm{~d}_{1}\right)}+\left\langle\mathrm{d}_{2} u, \mathrm{~d}_{2} u^{\prime}\right\rangle_{L^{2}\left(\mathrm{~d}_{2}\right)} \\
& +\left\langle\mathrm{d}_{3} u, \mathrm{~d}_{3} u^{\prime}\right\rangle_{L^{2}\left(\mathrm{~d}_{3}\right)},
\end{aligned}
$$

and

$$
\begin{aligned}
H\left(\mathrm{~d}_{1}, \mathrm{~d}_{2}, \mathrm{~d}_{3}, \mathrm{~d}_{4} ; \Omega\right):= & H\left(\mathrm{~d}_{1} ; \Omega\right) \cap H\left(\mathrm{~d}_{2} ; \Omega\right) \cap H\left(\mathrm{~d}_{3} ; \Omega\right) \cap H\left(\mathrm{~d}_{4} ; \Omega\right), \\
\left\langle u, u^{\prime}\right\rangle_{H\left(\mathrm{~d}_{1}, \mathrm{~d}_{2}, \mathrm{~d}_{3}, \mathrm{~d}_{4} ; \Omega\right)}:= & \left\langle u, u^{\prime}\right\rangle_{L^{2}(\Omega)}+\left\langle\mathrm{d}_{1} u, \mathrm{~d}_{1} u^{\prime}\right\rangle_{L^{2}\left(\mathrm{~d}_{1}\right)}+\left\langle\mathrm{d}_{2} u, \mathrm{~d}_{2} u^{\prime}\right\rangle_{L^{2}\left(\mathrm{~d}_{2}\right)} \\
& +\left\langle\mathrm{d}_{3} u, \mathrm{~d}_{3} u^{\prime}\right\rangle_{L^{2}\left(\mathrm{~d}_{3}\right)}+\left\langle\mathrm{d}_{4} u, \mathrm{~d}_{4} u^{\prime}\right\rangle_{L^{2}\left(\mathrm{~d}_{4}\right)} .
\end{aligned}
$$

\subsection{Assumptions on the domain and material parameters}

Assumption 2.1 (Assumption on $\epsilon$ ). Let $\epsilon \in\left(L^{\infty}(\Omega)\right)^{3 x 3}$ be so that there exists $c_{\epsilon}>0$ with

$$
c_{\epsilon}|\xi|^{2} \leq \Re\left(\xi^{H} \epsilon(x) \xi\right) \quad \text { and } \quad 0 \leq \Im\left(\xi^{H} \epsilon(x) \xi\right)
$$

for all $x \in \Omega$ and all $\xi \in \mathbb{C}^{3}$.

Assumption 2.2 (Assumption on $\mu$ ). Let $\mu^{-1} \in\left(L^{\infty}(\Omega)\right)^{3 x 3}$ be so that there exists $c_{\mu}>0$ with

$$
c_{\mu}|\xi|^{2} \leq \Re\left(\xi^{H} \mu^{-1}(x) \xi\right) \quad \text { and } \quad 0 \leq-\Im\left(\xi^{H} \mu^{-1}(x) \xi\right)
$$

for all $x \in \Omega$ and all $\xi \in \mathbb{C}^{3}$.

Assumption 2.3 (Assumption on $\Omega$ ). Let $\Omega \subset \mathbb{R}^{3}$ be a bounded path connected Lipschitz domain so that there exists $\delta>0$ and the following shift theorem holds on $\Omega$ : Let $f \in L^{2}(\Omega), g \in H^{1 / 2}(\partial \Omega)$ with $\langle f, 1\rangle_{L^{2}(\Omega)}+$ $\langle g, 1\rangle_{L^{2}(\partial \Omega)}=0$ and $w \in H_{*}^{1}(\Omega)$ be the solution to

$$
\begin{aligned}
-\Delta w=f \quad \text { in } \quad \Omega, \\
\nu \cdot \nabla w=g \quad \text { at } \quad \partial \Omega .
\end{aligned}
$$

Then the linear map $(f, g) \mapsto w: L^{2}(\Omega) \times H^{1 / 2}(\partial \Omega) \rightarrow H^{3 / 2+\delta}(\Omega)$ is well defined and continuous. 
The above assumption holds e.g. for smooth domains and Lipschitz polyhedral ([14], Cor. 23.5).

Assumption 2.4 (Assumption on $\Omega, \epsilon$ and $\mu^{-1}$ ). Let $\epsilon, \mu^{-1}$ and $\Omega$ be so that a unique continuation principle holds, i.e. if $u \in H(\operatorname{curl} ; \Omega)$ solves

$$
\begin{aligned}
& \operatorname{curl} \mu^{-1} \operatorname{curl} u-\omega^{2} \epsilon u=0 \quad \text { in } \Omega \text {, } \\
& \operatorname{tr}_{\nu \times} u=0 \text { at } \partial \Omega \text {, } \\
& \operatorname{tr}_{\nu \times} \mu^{-1} \operatorname{curl} u=0 \text { at } \partial \Omega \text {, }
\end{aligned}
$$

then $u=0$.

To our knowledge the most general todays available result on the unique continuation principle for Maxwell's equations is the one of Ball, Capdeboscq and Tsering-Xiao [4]. It essentially requires $\epsilon$ and $\mu^{-1}$ to be piece-wise $W^{1, \infty}$.

\subsection{Trace regularities and compact embeddings}

We recall some classical results on traces and embeddings, which will be essential for our analysis. We recall from Costabel [13]:

$$
\begin{aligned}
& \operatorname{tr}_{\nu .} \in L\left(H\left(\text { curl, div }, \operatorname{tr}_{\nu \times} ; \Omega\right), L^{2}(\partial \Omega)\right), \\
& \operatorname{tr}_{\nu \times} \in L\left(H\left(\text { curl, div, } \operatorname{tr}_{\nu} ; \Omega\right), \mathbf{L}_{t}^{2}(\partial \Omega)\right) .
\end{aligned}
$$

and

$$
\text { The embedding from } H\left(\operatorname{curl}, \operatorname{div}, \operatorname{tr}_{\nu \times} ; \Omega\right) \text { to } \mathbf{L}^{2}(\Omega) \text { is compact. }
$$

We deduce from Amrouche et al. ([1], Prop. 3.7):

If $\Omega$ satisfies Assumption 2.3, then $\operatorname{tr}_{\nu \times} \in L\left(H\left(\operatorname{curl}, \operatorname{div}, \operatorname{tr}_{\nu}^{0} ; \Omega\right), \mathbf{L}_{t}^{2}(\partial \Omega)\right)$ is compact.

\subsection{Helmholtz decomposition on the boundary}

We recall from Buffa et al. ([8], Thm. 5.5):

$$
\mathbf{L}_{t}^{2}(\partial \Omega)=\nabla_{\partial} H^{1}(\partial \Omega) \oplus^{\perp} \operatorname{curl}_{\partial} H^{1}(\partial \Omega)
$$

and denote the respective orthogonal projections by

$$
P_{\nabla_{\partial}}: \mathbf{L}_{t}^{2}(\partial \Omega) \rightarrow \nabla_{\partial} H^{1}(\partial \Omega), \quad P_{\nabla_{\partial}^{\top}}: \mathbf{L}_{t}^{2}(\partial \Omega) \rightarrow \operatorname{curl}_{\partial} H^{1}(\partial \Omega) .
$$

Recall $\operatorname{div}_{\partial} \operatorname{tr}_{\nu \times} \in L\left(H(\operatorname{curl} ; \Omega), H^{-1 / 2}(\partial \Omega)\right)$. So for $u \in H(\operatorname{curl} ; \Omega)$ let $z$ be the solution to find $z \in H_{*}^{1}(\partial \Omega)$ so that

$$
\left\langle\nabla_{\partial} z, \nabla_{\partial} z^{\prime}\right\rangle_{\mathbf{L}_{t}^{2}(\partial \Omega)}=-\left\langle\operatorname{div}_{\partial} \operatorname{tr}_{\nu \times} u, z^{\prime}\right\rangle_{H^{-1}(\partial \Omega) \times H^{1}(\partial \Omega)}
$$

for all $z^{\prime} \in H_{*}^{1}(\partial \Omega)$ and set

$$
S u:=\nabla_{\partial} z .
$$

From the construction of $S$ it follows $S \in L\left(H(\operatorname{curl} ; \Omega), \mathbf{L}_{t}^{2}(\partial \Omega)\right)$ and further

$$
S u=P_{\nabla \partial} \operatorname{tr}_{\nu \times} u
$$

for $u \in H\left(\right.$ curl, $\left.\operatorname{tr}_{\nu \times} ; \Omega\right)$. 


\section{Weak $T(\cdot)$-COERCIVITy of THE StekLOV Operator FUnCTION}

First we introduce the electromagnetic Steklov eigenvalue problem as holomorphic operator function eigenvalue problem. In Theorem 3.1 we report an apt decomposition of the respective Hilbert space into three subspaces. Next we introduce in (3.10) an operator function $T(\cdot)$ as an apt sign change on the subspaces. In Theorem 3.2 we report the weak $T(\cdot)$-coercivity of the Steklov operator function on $\mathbb{C} \backslash\{0\}$. In Corollary 3.3 we deduce convenient properties of the spectrum in $\mathbb{C} \backslash\{0\}$. In Corollary 3.4 we report that $\lambda=0$ constitutes the essential spectrum. However, these two Corollaries make no statement on the existence of eigenvalues. We report in a companion article [16] the existence and behavior of eigenvalues for purely real, symmetric $\mu$ and $\epsilon$, i.e. in the selfadjoint case.

Let $\omega>0$ be fixed. For $\lambda \in \mathbb{C}$ let $A(\lambda) \in L\left(H\left(\operatorname{curl}, \operatorname{tr}_{\nu \times} ; \Omega\right)\right)$ be defined through

$$
\begin{aligned}
\left\langle A(\lambda) u, u^{\prime}\right\rangle_{H\left(\operatorname{curl}, \operatorname{tr}_{\nu \times} ; \Omega\right):=} & \left\langle\mu^{-1} \operatorname{curl} u, \operatorname{curl} u^{\prime}\right\rangle_{\mathbf{L}^{2}(\Omega)}-\omega^{2}\left\langle\epsilon u, u^{\prime}\right\rangle_{\mathbf{L}^{2}(\Omega)} \\
& -\lambda\left\langle\operatorname{tr}_{\nu \times} u, \operatorname{tr}_{\nu \times} u^{\prime}\right\rangle_{\mathbf{L}_{t}^{2}(\partial \Omega)} \text { for all } u, u^{\prime} \in H\left(\operatorname{curl}, \operatorname{tr}_{\nu \times} ; \Omega\right) .
\end{aligned}
$$

The electromagnetic Steklov eigenvalue problem which we investigate in this section is to

$$
\text { find } \quad(\lambda, u) \in \mathbb{C} \times H\left(\operatorname{curl}, \operatorname{tr}_{\nu \times} ; \Omega\right) \backslash\{0\} \quad \text { so that } A(\lambda) u=0 .
$$

We note that the sign of $\lambda$ herein is reversed compared to [12]. To analyze the operator $A(\lambda)$ we introduce the following subspaces of $H\left(\operatorname{curl}, \operatorname{tr}_{\nu \times} ; \Omega\right)$ :

$$
\begin{aligned}
V & :=H\left(\operatorname{curl}, \operatorname{div}^{0}, \operatorname{tr}_{\nu \times}, \operatorname{tr}_{\nu}^{0} ; \Omega\right), \\
W_{1} & :=H\left(\operatorname{curl}^{0}, \operatorname{div}^{0}, \operatorname{tr}_{\nu \times} ; \Omega\right), \\
W_{2} & :=\nabla H_{0}^{1} \subset H\left(\operatorname{curl}^{0}, \operatorname{tr}_{\nu \times}^{0} ; \Omega\right) .
\end{aligned}
$$

Theorem 3.1. It holds

$$
H\left(\operatorname{curl}, \operatorname{tr}_{\nu \times} ; \Omega\right)=\left(V \oplus W_{1}\right) \oplus^{\perp_{H\left(\operatorname{curl}_{1}, \mathrm{tr}_{\nu \times} ; \Omega\right)}} W_{2}
$$

in the following sense. There exist projections $P_{V}, P_{W_{1}}, P_{W_{2}} \in L\left(H\left(\operatorname{curl}, \operatorname{tr}_{\nu \times} ; \Omega\right)\right)$ with $\operatorname{ran} P_{V}=V, \operatorname{ran} P_{W_{1}}=$ $W_{1}, \operatorname{ran} P_{W_{2}}=W_{2}, W_{1}, W_{2} \subset \operatorname{ker} P_{V}, V, W_{2} \subset \operatorname{ker} P_{W_{1}}, V, W_{1} \subset \operatorname{ker} P_{W_{2}}$ and $u=P_{v} u+P_{W_{1}} u+P_{W_{2}} u$ for each $u \in H\left(\operatorname{curl}, \operatorname{tr}_{\nu \times} ; \Omega\right)$. Thus, the norm induced by

$$
\begin{aligned}
\left\langle u, u^{\prime}\right\rangle_{X}:= & \left\langle P_{V} u, P_{V} u^{\prime}\right\rangle_{H\left(\operatorname{curl}, \operatorname{tr}_{\nu \times} ; \Omega\right)}+\left\langle P_{W_{1}} u, P_{W_{1}} u^{\prime}\right\rangle_{H\left(\operatorname{curl}_{1}, \operatorname{tr}_{\nu \times} ; \Omega\right)} \\
& +\left\langle P_{W_{2}} u, P_{W_{2}} u^{\prime}\right\rangle_{H\left(\operatorname{curl}_{1}, \operatorname{tr}_{\nu \times} ; \Omega\right)}, \quad u, u^{\prime} \in H\left(\operatorname{curl}, \operatorname{tr}_{\nu \times} ; \Omega\right)
\end{aligned}
$$

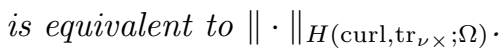

Proof. Step 1. Let $P_{W_{2}}$ be the $H\left(\operatorname{curl}, \operatorname{tr}_{\nu \times} ; \Omega\right)$-orthogonal projection onto $W_{2}$. Hence $P_{W_{2}} \in$ $L\left(H\left(\operatorname{curl}, \operatorname{tr}_{\nu \times} ; \Omega\right)\right)$ is a projection with range $W_{2}$ and kernel

$$
W_{2}^{\perp_{H\left(\mathrm{curl}_{\mathrm{tr}} \times \mathrm{i} ; \Omega\right)}}=H\left(\mathrm{curl}, \operatorname{div}^{0}, \operatorname{tr}_{\nu \times} ; \Omega\right) \supset V, W_{1} .
$$

Step 2a. Let $u \in H\left(\operatorname{curl}, \operatorname{tr}_{\nu \times} ; \Omega\right)$. Note that due to $\operatorname{div}\left(u-P_{W_{2}} u\right)=0$ and $(2.7)$ it holds $\operatorname{tr}_{\nu \cdot}\left(u-P_{W_{2}} u\right) \in$ $L^{2}(\partial \Omega)$ and $\left\langle\operatorname{tr}_{\nu \cdot}\left(u-P_{W_{2}} u\right), 1\right\rangle_{L^{2}(\partial \Omega)}=0$. Let $w_{*} \in H_{*}^{1}(\Omega)$ be the unique solution to

$$
-\Delta w_{*}=0 \quad \text { in } \Omega, \quad \nu \cdot \nabla w_{*}=\operatorname{tr}_{\nu \cdot}\left(u-P_{W_{2}} u\right) \text { at } \partial \Omega .
$$

Let $P_{W_{1}} u:=\nabla w_{*}$. By construction of $P_{W_{1}}$ and due to (2.7) it holds $\operatorname{ran} P_{W_{1}} \subset W_{1}$ and $P_{W_{1}} \in$ $L\left(H\left(\operatorname{curl}, \operatorname{tr}_{\nu \times} ; \Omega\right)\right)$. Let $u \in W_{1}$. Then $P_{W_{2}} u=0$ and hence $P_{W_{1}} u=u$. Thus $P_{W_{1}}$ is a projection and $\operatorname{ran} P_{W_{1}}=W_{1}$. 
Step 2b. If $u \in W_{2}$ then $u-P_{W_{2}} u=0$, further $\operatorname{tr}_{\nu} \cdot\left(u-P_{W_{2}} u\right)=0$ and thus $P_{W_{1}} u=0$. Hence $W_{2} \subset \operatorname{ker} P_{W_{1}}$. If $u \in V$ then $P_{W_{2}} u=0$, further $\operatorname{tr}_{\nu} \cdot\left(u-P_{W_{2}} u\right)=\operatorname{tr}_{\nu} . u=0$ and thus $P_{W_{1}} u=0$. Hence $V \subset \operatorname{ker} P_{W_{1}}$.

Step 3. Let $u \in H\left(\operatorname{curl}, \operatorname{tr}_{\nu \times} ; \Omega\right)$ and $P_{V} u:=u-P_{W_{1}} u-P_{W_{2}} u$. It follow $P_{V} \in L\left(H\left(\right.\right.$ curl, $\left.\left.\operatorname{tr}_{\nu \times} ; \Omega\right)\right), P_{V} u \in V$ and $P_{V} P_{V} u=P_{V} u$, i.e. $P_{V}$ is a bounded projection. If $u \in V$ then $P_{W_{1}} u=P_{W_{2}} u=0$ and thus $P_{V} u=u$. Hence ran $P_{V}=V$. It follows further $W_{1}, W_{2} \subset \operatorname{ker} P_{V}$.

Step 4. By means of the triangle inequality and a Young inequality it holds

$$
\begin{aligned}
\|u\|_{H\left(\operatorname{curl}, \operatorname{tr}_{\nu \times} ; \Omega\right)}^{2} & =\left\|P_{V} u+P_{W_{1}} u+P_{W_{2}} u\right\|_{H\left(\operatorname{curl}_{\left.1 \operatorname{tr}_{\nu \times} ; \Omega\right)}\right.}^{2} \\
& \leq 3\left(\left\|P_{V} u\right\|_{H\left(\operatorname{curl}, \operatorname{tr}_{\nu \times} ; \Omega\right)}^{2}+\left\|P_{W_{1}} u\right\|_{H\left(\operatorname{curl}, \operatorname{tr}_{\nu \times} ; \Omega\right)}^{2}+\left\|P_{W_{2}} u\right\|_{H\left(\operatorname{curl}, \operatorname{tr}_{\nu \times} ; \Omega\right)}^{2}\right) \\
& =3\|u\|_{X}^{2} .
\end{aligned}
$$

On the other hand due to the boundedness of the projections

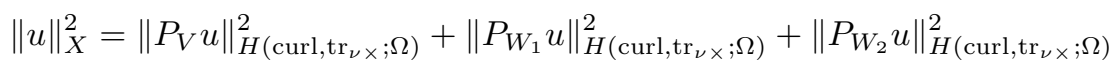

$$
\begin{aligned}
& \leq\left(\left\|P_{V}\right\|_{L\left(H\left(\operatorname{curl}, \operatorname{tr}_{\nu \times} ; \Omega\right)\right)}^{2}+\left\|P_{W_{1}}\right\|_{L\left(H\left(\operatorname{curl}, \operatorname{tr}_{\nu \times} ; \Omega\right)\right)}^{2}\right. \\
& +\left\|P_{W_{2}}\right\|_{L\left(H\left(\operatorname{curl}_{\left.\left.1, \operatorname{tr}_{\nu \times} ; \Omega\right)\right)}^{2}\right)\right.}^{2}\|u\|_{H\left(\operatorname{curl}_{\left.1, \operatorname{tr}_{\nu \times} ; \Omega\right)}^{2}\right.}^{2} .
\end{aligned}
$$

Let us look at $A(\lambda)$ in light of this substructure of $H\left(\operatorname{curl}, \operatorname{tr}_{\nu \times} ; \Omega\right)$. To this end we consider the space

$$
X:=H\left(\operatorname{curl}, \operatorname{tr}_{\nu \times} ; \Omega\right), \quad\langle\cdot, \cdot\rangle_{X} \quad \text { as defined in }(3.5) .
$$

It follows that $P_{V}, P_{W_{1}}$ and $P_{W_{1}}$ are even orthogonal projections in $X$. Let further $A_{X}(\cdot), A_{c}, A_{\epsilon}, A_{l^{2}}, A_{\mathrm{tr}} \in L(X)$ be defined through

$$
\begin{aligned}
\left\langle A_{X}(\lambda) u, u^{\prime}\right\rangle_{X} & :=\left\langle A(\lambda) u, u^{\prime}\right\rangle_{H\left(\operatorname{curl}, \operatorname{tr}_{\nu \times} ; \Omega\right)} & & \text { for all } u, u^{\prime} \in X, \lambda \in \mathbb{C} \\
\left\langle A_{c} u, u^{\prime}\right\rangle_{X} & :=\left\langle\mu^{-1} \operatorname{curl} u, \operatorname{curl} u^{\prime}\right\rangle_{\mathbf{L}^{2}(\Omega)} & & \text { for all } u, u^{\prime} \in X, \\
\left\langle A_{\epsilon} u, u^{\prime}\right\rangle_{X} & :=\left\langle\epsilon u, u^{\prime}\right\rangle_{\mathbf{L}^{2}(\Omega)} & & \text { for all } u, u^{\prime} \in X, \\
\left\langle A_{l^{2}} u, u^{\prime}\right\rangle_{X} & :=\left\langle u, u^{\prime}\right\rangle_{\mathbf{L}^{2}(\Omega)} & & \text { for all } u, u^{\prime} \in X, \\
\left\langle A_{\operatorname{tr}} u, u^{\prime}\right\rangle_{X} & :=\left\langle\operatorname{tr}_{\nu \times} u, \operatorname{tr}_{\nu \times} u^{\prime}\right\rangle_{\mathbf{L}_{t}^{2}(\partial \Omega)} & & \text { for all } u, u^{\prime} \in X .
\end{aligned}
$$

We deduce from the definitions of $V, W_{1}$ and $W_{2}$ that

$$
\begin{aligned}
A_{X}(\lambda)= & \left(P_{V}+P_{W_{1}}+P_{W_{2}}\right)\left(A_{c}-\omega^{2} A_{\epsilon}-\lambda A_{\mathrm{tr}}\right)\left(P_{V}+P_{W_{1}}+P_{W_{2}}\right) \\
= & P_{V} A_{c} P_{V}-\omega^{2}\left(P_{V}+P_{W_{1}}+P_{W_{2}}\right) A_{\epsilon}\left(P_{V}+P_{W_{1}}+P_{W_{2}}\right) \\
& -\lambda\left(P_{V}+P_{W_{1}}\right) A_{\mathrm{tr}}\left(P_{V}+P_{W_{1}}\right) \\
= & \mathbf{P}_{\mathbf{V}} \mathbf{A}_{\mathbf{c}} \mathbf{P}_{\mathbf{V}}-\omega^{2} \mathbf{P}_{\mathbf{W}_{\mathbf{2}}} \mathbf{A}_{\epsilon} \mathbf{P}_{\mathbf{W}_{\mathbf{2}}}-\lambda \mathbf{P}_{\mathbf{W}_{1}} \mathbf{A}_{\mathrm{tr}} \mathbf{P}_{\mathbf{W}_{1}} \\
& -\omega^{2}\left(P_{V} A_{\epsilon} P_{V}+P_{W_{1}} A_{\epsilon} P_{W_{1}}\right) \\
& -\lambda\left(P_{V} A_{\mathrm{tr}} P_{V}+P_{V} A_{\mathrm{tr}} P_{W_{1}}+P_{W_{1}} A_{\mathrm{tr}} P_{V}\right) .
\end{aligned}
$$

If we identify $X \sim V \times W_{1} \times W_{2}$ and $X \ni u \sim\left(v, w_{1}, w_{2}\right) \in V \times W_{1} \times W_{2}$, we can identify $A_{X}(\lambda)$ with the block operator

$$
\left(\begin{array}{ccc}
\left.\mathbf{P}_{\mathbf{V}} \mathbf{A}_{\mathbf{c}}\right|_{\mathbf{V}}-\left.P_{V}\left(\omega^{2} A_{\epsilon}+\lambda A_{\mathrm{tr}}\right)\right|_{V} & -\left.P_{V}\left(\omega^{2} A_{\epsilon}+\lambda A_{\mathrm{tr}}\right)\right|_{W_{1}} & -\left.\omega^{2} P_{V} A_{\epsilon}\right|_{W_{2}} \\
-\left.P_{W_{1}}\left(\omega^{2} A_{\epsilon}+\lambda A_{\mathrm{tr}}\right)\right|_{V} & -\omega^{2} P_{W_{1}} A_{\epsilon}\left|W_{1}-\lambda \mathbf{P}_{\mathbf{W}_{1}} \mathbf{A}_{\mathrm{tr}}\right| \mathbf{W}_{\mathbf{1}} & -\left.\omega^{2} P_{W_{1}} A_{\epsilon}\right|_{W_{2}} \\
-\left.\omega^{2} P_{W_{2}} A_{\epsilon}\right|_{V} & -\left.\omega^{2} P_{W_{2}} A_{\epsilon}\right|_{W_{1}} & -\left.\omega^{\mathbf{2}} \mathbf{P}_{\mathbf{W}_{\mathbf{2}}} \mathbf{A}_{\epsilon}\right|_{\mathbf{W}_{\mathbf{2}}}
\end{array}\right) .
$$


We highlighted with color and boldface in (3.8) and (3.9) the operators which are not compact. This leads us to define a test function operator function in the following way. Let

$$
T(\lambda):=P_{V}-\bar{\lambda}^{-1} P_{W_{1}}-\omega^{-2} P_{W_{2}}, \quad \lambda \in \mathbb{C} \backslash\{0\} .
$$

Obviously $T(\lambda) \in L(X)$ is bijective with $T(\lambda)^{-1}=P_{V}-\bar{\lambda} P_{W_{1}}-\omega^{2} P_{W_{2}}$ for $\lambda \in \mathbb{C} \backslash\{0\}$.

Theorem 3.2. Let $\epsilon$ satisfy Assumption 2.1, $\mu$ satisfy Assumption 2.2 and $\Omega$ satisfy Assumption 2.3. Thence $A_{X}(\cdot): \mathbb{C} \backslash\{0\} \rightarrow L(X)$ is weakly $T(\cdot)$-coercive.

Proof. Let $\lambda \in \mathbb{C} \backslash\{0\}$. Let

$$
\begin{aligned}
A_{1}:= & P_{V} A_{c} P_{V}+P_{V} A_{l^{2}} P_{V}+P_{V} A_{\mathrm{tr}} P_{V} \\
& -\lambda P_{W_{1}} A_{l^{2}} P_{W_{1}}-\lambda P_{W_{1}} A_{\mathrm{tr}} P_{W_{1}}-\omega^{2} P_{W_{2}} A_{\epsilon} P_{W_{2}}
\end{aligned}
$$

and

$$
\begin{aligned}
A_{2}:= & -\omega^{2}\left(P_{V} A_{\epsilon} P_{V}+P_{W_{1}} A_{\epsilon} P_{W_{1}}+P_{V} A_{\epsilon} P_{W_{1}}+P_{W_{1}} A_{\epsilon} P_{V}\right. \\
& \left.+P_{V} A_{\epsilon} P_{W_{2}}+P_{W_{2}} A_{\epsilon} P_{V}+P_{W_{1}} A_{\epsilon} P_{W_{2}}+P_{W_{2}} A_{\epsilon} P_{W_{1}}\right) \\
& -P_{V} A_{l^{2}} P_{V}-(1+\lambda) P_{V} A_{\mathrm{tr}} P_{V} \\
& +\lambda P_{W_{1}} A_{l^{2}} P_{W_{1}}-\lambda\left(P_{V} A_{\mathrm{tr}} P_{W_{1}}+P_{W_{1}} A_{\mathrm{tr}} P_{V}\right) .
\end{aligned}
$$

so that $A_{X}(\lambda)=A_{1}+A_{2}$. Operator $A_{2}$ is compact due to (2.8) and (2.9) and hence so is $T^{*} A_{2}$. It is straight forward to see

$$
\Re\left(\left\langle A_{1} u, T(\lambda) u\right\rangle_{X}\right) \geq \min \left(1, c_{\epsilon}, c_{\mu}\right)\|u\|_{X}^{2},
$$

i.e. $T(\lambda)^{*} A_{1}$ is coercive.

We remark that the naming of the (sub)spaces as $X, V, W_{1}, W_{2}$ follows Buffa [7] while the naming of the "test function operator" as $T(\lambda)$ follows e.g. Bonnet-Ben Dhia, Ciarlet and Zwölf [6].

Corollary 3.3. Let Assumptions 2.1-2.4 hold true. Then $A_{X}(\lambda)$ is bijective for all $\lambda \in \mathbb{C}$ with $\Im(\lambda)<0$. Hence the spectrum of $A_{X}(\cdot)$ in $\mathbb{C} \backslash\{0\}$ consists of an at most countable set of eigenvalues with finite algebraic multiplicity which has no accumulation point in $\mathbb{C} \backslash\{0\}$.

Proof. Let $\lambda \in \mathbb{C}$ with $\Im(\lambda)<0$ and $u \in X$ be so that $A_{X}(\lambda) u=0$. It follows

$$
\left.0=-\Im\left(\left\langle A_{X}(\lambda) u, u\right)\right\rangle_{X}\right) \geq-\Im(\lambda)\left\|\operatorname{tr}_{\nu \times} u\right\|_{\mathbf{L}_{t}^{2}(\partial \Omega)}^{2}
$$

and together with Assumption 2.4 it follows further $u=0$, i.e. $A_{X}(\lambda)$ is injective. From Theorem 3.2 we know that $A_{X}(\lambda)$ is Fredholm with index zero for all $\lambda \in \mathbb{C} \backslash\{0\}$ and hence $A_{X}(\lambda)$ is bijective, if $\Im(\lambda)<0$.

Further $A_{X}(\cdot)$ is holomorphic since it is even an affine function. The resolvent set of $A_{X}(\cdot): \mathbb{C} \backslash\{0\} \rightarrow L(X)$ is non-empty. The result on the spectrum in $\mathbb{C} \backslash\{0\}$ is a classical result on holomorphic Fredholm operator functions, see e.g. Proposition A.8.4 of [20].

Corollary 3.4. Let $\epsilon$ satisfy Assumptions 2.1. Then $A_{X}(0)$ is not Fredholm.

Proof. We construct a singular sequence $\left(w_{1, n} \in W_{1}\right)_{n \in \mathbb{N}}$ for $A(0)$, i.e. $\left\|w_{1, n}\right\|_{X}=1$ for each $n \in \mathbb{N},\left(w_{1, n}\right)_{n \in \mathbb{N}}$ admits no converging subsequence and $\lim _{n \in \mathbb{N}} A(0) w_{1, n}=0$.

To this end let $\left(f_{n} \in L^{2}(\partial \Omega) \backslash\{0\}\right)_{n \in \mathbb{N}}$ be a sequence with $\left\langle f_{n}, 1\right\rangle_{L^{2}(\partial \Omega)}=0$ for each $n \in \mathbb{N}$ and which admits no converging subsequence in $L^{2}(\partial \Omega)$, but which converges to $f \in H^{-1 / 2}(\partial \Omega) \backslash L^{2}(\partial \Omega)$ in $H^{-1 / 2}(\partial \Omega)$ so that $\left\|f_{n}\right\|_{L^{2}(\partial \Omega)} \rightarrow+\infty$ as $n \rightarrow+\infty$. Let $\tilde{w}_{1, n} \in H_{*}^{1}(\Omega)$ be the solution to

$$
\begin{array}{cl}
-\Delta \tilde{w}_{1, n}=0 & \text { in } \Omega, \\
\nu \cdot \nabla \tilde{w}_{1, n}=f_{n} & \text { at } \partial \Omega .
\end{array}
$$


The volume part of the norm $\left\|\nabla \tilde{w}_{1, n}\right\|_{\mathbf{L}^{2}(\Omega)}$ can be uniformly bounded by

$$
\sup _{n \in \mathbb{N}}\left\|f_{n}\right\|_{H^{-1 / 2}(\partial \Omega)}
$$

Due to (2.7) we know that there exists $C>0$ independent of $\nabla \tilde{w}_{1, n}$ so that

$$
\left\|f_{n}\right\|_{L^{2}(\partial \Omega)}=\left\|\operatorname{tr}_{\nu} . \nabla \tilde{w}_{1, n}\right\|_{L^{2}(\partial \Omega)} \leq C\left(\left\|\nabla \tilde{w}_{1, n}\right\|_{\mathbf{L}^{2}(\Omega)}+\left\|\operatorname{tr}_{\nu \times} \nabla \tilde{w}_{1, n}\right\|_{\mathbf{L}_{t}^{2}(\partial \Omega)}\right) .
$$

It follows $\left\|\operatorname{tr}_{\nu \times} \nabla \tilde{w}_{1, n}\right\|_{\mathbf{L}_{t}^{2}(\partial \Omega)} \rightarrow+\infty$ as $n \rightarrow+\infty$. Hence

$$
\left\|A_{X}(0) \nabla \tilde{w}_{1, n}\right\|_{X} \leq \sqrt{3}\|\epsilon\|_{\left(L^{\infty}(\Omega)\right)^{3 \times 3}}\left\|\nabla \tilde{w}_{1, n}\right\|_{\mathbf{L}^{2}(\Omega)} .
$$

Let $w_{1, n}:=\nabla \tilde{w}_{1, n} /\left\|\nabla \tilde{w}_{1, n}\right\|_{X}$. It follows $\left\|w_{1, n}\right\|_{X}=1$ and $A_{X}(0) w_{1, n} \rightarrow 0$ as $n \rightarrow+\infty$. The existence of a converging subsequence of $\left(w_{1, n} \in W_{1}\right)_{n \in \mathbb{N}}$ would imply that $\left(f_{n} \in L^{2}(\partial \Omega)\right)_{n \in \mathbb{N}}$ admits a converging subsequence, which is a contradiction. Hence $\left(w_{1, n}\right)_{n \in \mathbb{N}}$ is indeed a singular sequence for $A_{X}(0)$.

\section{Compatible approximation of the Steklov eigenvalue problem}

In this section we discuss Galerkin approximations of (3.2). In addition to the basic Assumption 4.1 we embrace in Assumption 4.2 the existence of uniformly bounded commuting projections like in [2]. Since we work with the space $H\left(\operatorname{curl}, \operatorname{tr}_{\nu \times} ; \Omega\right)$ rather than $H(\operatorname{curl} ; \Omega)$, our assumption concerns an additional projection on $\mathbf{L}_{t}^{2}(\partial \Omega)$ compared to [2]. We report in Corollary 4.5 that for Galerkin approximations which satisfy these two assumptions, we can construct a sequence of operator functions $T_{n}(\cdot): \mathbb{C} \backslash\{0\} \rightarrow L\left(X_{n}\right)$ which converges to $T(\cdot)$ in discrete norm (2.1) at each $\lambda \in \mathbb{C} \backslash\{0\}$. The proof is based on Lemmas 4.3 and 4.4 and applies techniques as outlined in [2]. Consequently we report in Theorem 4.6 that the abstract framework of [17] (which is based on the exhaustive works of Karma $[18,19]$ ) is applicable. However, the existence and possible construction of such projection operators remain open questions!

Consider the following basic assumption.

Assumption 4.1. Let $\left(X_{n}\right)_{n \in \mathbb{N}}$ be so that $X_{n} \subset X$ and $\operatorname{dim} X_{n}<\infty$ for each $n \in \mathbb{N}$, and

$$
\lim _{n \in \mathbb{N}} \inf _{u^{\prime} \in X_{n}}\left\|u-u^{\prime}\right\|_{X}=0 \text { for each } u \in X
$$

Consider the following additional assumption.

Assumption 4.2. There exists $\left(\pi_{n}^{X}\right)_{n \in \mathbb{N}}$ so that

$$
\begin{array}{r}
\pi_{n}^{X} \in L\left(\mathbf{L}^{2}(\Omega)\right) \text { is a projector with } X_{n}=\operatorname{ran} \pi_{n}^{X}, \\
\qquad \sup _{n \in \mathbb{N}}\left\|\pi_{n}^{X}\right\|_{L\left(\mathbf{L}^{2}(\Omega)\right)}<+\infty .
\end{array}
$$

Let $Y:=\mathbf{L}^{2}(\Omega)$ and $Z:=\mathbf{L}_{t}^{2}(\partial \Omega)$. There exist sequences $\left(Y_{n}, Z_{n}, \pi_{n}^{Y}, \pi_{n}^{Z},\right)_{n \in \mathbb{N}}$ so that for each $H \in\{Y, Z\}$ it holds

$$
\begin{array}{r}
H_{n} \subset H, \quad \lim _{n \in \mathbb{N}} \inf _{u^{\prime} \in H_{n}}\left\|u-u^{\prime}\right\|_{H}=0, \\
\pi_{n}^{H} \in L(H) \text { is a projector with } H_{n} \subset \operatorname{ran} \pi_{n}^{H}, \\
\sup _{n \in \mathbb{N}}\left\|\pi_{n}^{H}\right\|_{L(H)}<+\infty .
\end{array}
$$

Denote $E \in L\left(X, \mathbf{L}^{2}(\Omega)\right)$ the embedding operator and set

$$
\pi_{n}:=\pi_{n}^{X} E .
$$


Further let

$$
\operatorname{curl} \circ \pi_{n} u=\pi_{n}^{Y} \circ \operatorname{curl} u \quad \text { and } \quad \operatorname{tr}_{\nu \times} \circ \pi_{n} u=\pi_{n}^{Z} \circ \operatorname{tr}_{\nu \times} u
$$

for each $u \in X$.

Lemma 4.3. Let Assumptions 4.1 and 4.2 hold true. Then the projections $\pi_{n}^{X}, \pi_{n}^{Y}$ and $\pi_{n}^{Z}$ converge point-wise to the identity in $\mathbf{L}^{2}(\Omega), \mathbf{L}^{2}(\Omega)$ and $\mathbf{L}_{t}^{2}(\partial \Omega)$ respectively.

Proof. We proceed as in [2]. Let $u \in \mathbf{L}^{2}(\Omega)$ and $u_{n} \in X_{n}$. Since $\pi_{n}^{X}$ is a projector it follows

$$
\begin{aligned}
\left\|\left(1-\pi_{n}^{X}\right) u\right\|_{\mathbf{L}^{2}(\Omega)} & =\left\|\left(1-\pi_{n}^{X}\right)\left(u-u_{n}\right)\right\|_{\mathbf{L}^{2}(\Omega)} \\
& \leq\left(1+\sup _{n \in \mathbb{N}}\left\|\pi_{n}^{X}\right\|_{L\left(\mathbf{L}^{2}(\Omega)\right)}\right)\left\|u-u_{n}\right\|_{\mathbf{L}^{2}(\Omega)}
\end{aligned}
$$

and hence

$$
\left\|\left(1-\pi_{n}^{X}\right) u\right\|_{\mathbf{L}^{2}(\Omega)} \leq\left(1+\sup _{n \in \mathbb{N}}\left\|\pi_{n}^{X}\right\|_{L\left(\mathbf{L}^{2}(\Omega)\right)}\right) \inf _{u_{n} \in X_{n}}\left\|u-u_{n}\right\|_{\mathbf{L}^{2}(\Omega)} .
$$

Since $X$ is densely embedded in $\mathbf{L}^{2}(\Omega)$ and due to Assumption 4.1 the claim follows for $\pi_{n}^{X}$. The claims for $\pi_{n}^{Y}$ and $\pi_{n}^{Z}$ follow like-wise.

Lemma 4.4. Let Assumptions 2.1, 2.3, 4.1 and 4.2 hold true. Then

$$
\begin{aligned}
& \lim _{n \in \mathbb{N}} \inf _{u \in X_{n} \backslash\{0\}}\left\|\left(1-\pi_{n}\right) P_{V} u\right\|_{X} /\|u\|_{X}=0, \\
& \lim _{n \in \mathbb{N}} \inf _{u \in X_{n} \backslash\{0\}}\left\|\left(1-\pi_{n}\right) P_{W_{1}} u\right\|_{X} /\|u\|_{X}=0, \\
& \lim _{n \in \mathbb{N}} \inf _{u \in X_{n} \backslash\{0\}}\left\|\left(1-\pi_{n}\right) P_{W_{2}} u\right\|_{X} /\|u\|_{X}=0 .
\end{aligned}
$$

Proof. We proceed as in [2]. Let $u_{n} \in X_{n}$. Due to curl $P_{W_{2}} u_{n}=0, \operatorname{tr}_{\nu \times} P_{W_{2}} u_{n}=0$ and Assumption 4.2 it hold

$$
\operatorname{curl} \pi_{n} P_{W_{2}} u_{n}=\pi_{n}^{Y} \operatorname{curl} P_{W_{2}} u_{n}=0
$$

and

$$
\operatorname{tr}_{\nu \times} \pi_{n} P_{W_{2}} u_{n}=\pi_{n}^{Z} \operatorname{tr}_{\nu \times} P_{W_{2}} u_{n}=0
$$

Hence

$$
\begin{aligned}
\left\|\left(1-\pi_{n}\right) P_{W_{2}} u_{n}\right\|_{X} & =\left\|\left(1-\pi_{n}\right) P_{W_{2}} u_{n}\right\|_{\mathbf{L}^{2}(\Omega)}=\left\|\left(1-\pi_{n}\right)\left(1-P_{W_{2}}\right) u_{n}\right\|_{\mathbf{L}^{2}(\Omega)} \\
& \leq\left\|\left(1-\pi_{n}^{X}\right) E\left(1-P_{W_{2}}\right)\right\|_{L\left(X, \mathbf{L}^{2}(\Omega)\right)}\left\|u_{n}\right\|_{X} .
\end{aligned}
$$

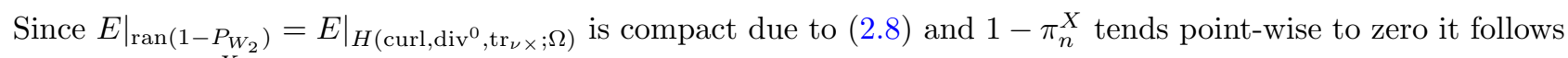
$\lim _{n \in \mathbb{N}}\left\|\left(1-\pi_{n}^{X}\right) E\left(1-P_{W_{2}}\right)\right\|_{L\left(X, \mathbf{L}^{2}(\Omega)\right)}=0$.

We compute

$$
\begin{aligned}
\operatorname{curl} \pi_{n} P_{V} u_{n} & =\pi_{n}^{Y} \operatorname{curl} P_{V} u_{n}=\pi_{n}^{Y} \operatorname{curl}\left(P_{V}+P_{W_{1}}+P_{W_{2}}\right) u_{n} \\
& =\pi_{n}^{Y} \operatorname{curl} u_{n}=\operatorname{curl} u_{n}=\operatorname{curl}\left(P_{V}+P_{W_{1}}+P_{W_{2}}\right) u_{n}=\operatorname{curl} P_{V} u_{n}
\end{aligned}
$$

and hence

$$
\left\|\left(1-\pi_{n}\right) P_{V} u_{n}\right\|_{X}^{2}=\left\|\left(1-\pi_{n}\right) P_{V} u_{n}\right\|_{\mathbf{L}^{2}(\Omega)}^{2}+\left\|\operatorname{tr}_{\nu \times}\left(1-\pi_{n}\right) P_{V} u_{n}\right\|_{\mathbf{L}_{t}^{2}(\partial \Omega)}^{2} .
$$

We estimate the first term

$$
\left\|\left(1-\pi_{n}\right) P_{V} u_{n}\right\|_{\mathbf{L}^{2}(\Omega)} \leq\left\|\left(1-\pi_{n}^{X}\right) E P_{V}\right\|_{L\left(X, \mathbf{L}^{2}(\Omega)\right)}\left\|u_{n}\right\|_{X} .
$$


As previously we obtain $\lim _{n \in \mathbb{N}}\left\|\left(1-\pi_{n}^{X}\right) E P_{V}\right\|_{L\left(X, \mathbf{L}^{2}(\Omega)\right)}=0$. We estimate the second term

$$
\begin{aligned}
\left\|\operatorname{tr}_{\nu \times}\left(1-\pi_{n}\right) P_{V} u_{n}\right\|_{\mathbf{L}_{t}^{2}(\partial \Omega)} & =\left\|\left(1-\pi_{n}^{Z}\right) \operatorname{tr}_{\nu \times} P_{V} u_{n}\right\|_{\mathbf{L}_{t}^{2}(\partial \Omega)} \\
& \leq\left\|\left(1-\pi_{n}^{Z}\right) \operatorname{tr}_{\nu \times} P_{V}\right\|_{L\left(X, \mathbf{L}_{t}^{2}(\partial \Omega)\right)}\left\|u_{n}\right\|_{X} .
\end{aligned}
$$

Due to (2.9) $\left.\operatorname{tr}_{\nu \times}\right|_{V}$ is compact, $\left(1-\pi_{n}^{Z}\right)$ tends point-wise to zero and hence

$$
\lim _{n \in \mathbb{N}}\left\|\left(1-\pi_{n}^{Z}\right) \operatorname{tr}_{\nu \times} P_{V}\right\|_{L\left(X, \mathbf{L}_{t}^{2}(\partial \Omega)\right)}=0 .
$$

The claim for $P_{W_{1}}$ follows from $P_{W_{1}}=1-P_{V}-P_{W_{2}}$.

Corollary 4.5. Let Assumptions 2.1, 2.3, 4.1 and 4.2 hold true. Let $T_{n}(\lambda) \in L\left(X_{n}\right)$ be defined as $T_{n}(\lambda):=$ $\left.\pi_{n} T(\lambda)\right|_{X_{n}}$ for each $\lambda \in \mathbb{C} \backslash\{0\}$. Then

$$
\lim _{n \in \mathbb{N}}\left\|T(\lambda)-T_{n}(\lambda)\right\|_{n}=0
$$

for each $\lambda \in \mathbb{C} \backslash\{0\}$.

Proof. Follows from the definition of $T(\lambda)$, the triangle inequality and Lemma 4.4.

Theorem 4.6. Let Assumptions 2.1-2.4 hold true. Let $X, A_{X}(\cdot)$ and $T(\cdot)$ be as defined in (3.6), (3.7a) and (3.10) respectively. Let Assumptions 4.1 and 4.2 hold true. Then $A_{X}(\cdot): \mathbb{C} \backslash\{0\} \rightarrow L(X)$ is a holomorphic weakly $T(\cdot)$-coercive operator function with non-empty resolvent set and the sequence of Galerkin approximations $\left(\left.P_{n} A_{X}(\cdot)\right|_{X_{n}}: \mathbb{C} \backslash\{0\} \rightarrow L\left(X_{n}\right)\right)_{n \in \mathbb{N}}$ is $T(\cdot)$-compatible.

Thus Corollary 2.8 of [17] is applicable and the convergence statements (i)-(vii) formulated in Subsection 2.1 hold.

Proof. Follows from Theorem 3.2, Corollaries 3.3 and 4.5.

The consequence of Theorem 4.6 is that if we approximate Eigenvalue Problem (3.2) with finite element methods which satisfy Assumptions 4.1 and 4.2, then we obtain asymptotically reliable approximations. However, the second commutation in (4.5) is of no concern in the literature on the design and analysis of common $H$ (curl)finite element spaces (e.g. [2]). Thus it is unclear if those spaces satisfy Assumption 4.2 as welcome byproduct and produce converging methods. Further numerical studies are necessary to determine if this is the case or not. If common $H$ (curl)-finite element methods fail, then our previous presented analysis may raise ideas for the construction of new suitable elements.

On the other hand, if common $H$ (curl)-finite element methods work, then it is likely that Assumption 4.2 is satisfied.

If the existence of such commuting projection operators would be proven, then this would complete our presented convergence analysis. However, the construction and analysis of commuting projection operators is an intricate topic of its own: We recall that the natural interpolation operators commute with the projection operators. Though, the interpolation operators are only well-defined for smooth enough functions. It is possible to couple the interpolation with suitable smoothing operators to achieve well-defined and bounded commuting projection operators (see e.g. [15]). If we consider the natural interpolation operators again, we note that they already fulfill the second commutation in (4.5). Hence the construction of suitable smoothing operators (which also respect the second commutation in (4.5)) would yield the desired result.

We emphasize that these commuting projection operators are a theoretical tool to conduct the analysis and are not required for the actual implementation of the finite element method.

For the former reasons the presented result is a bit unsatisfactory and further research is necessary. However, in the next two sections we deal with a modified eigenvalue problem for which we can report more rigorous results. 


\section{Weak $T(\cdot)$-Coercivity of the modified Steklov operator function}

First we introduce the modified electromagnetic Steklov eigenvalue problem proposed in [12] as holomorphic operator function eigenvalue problem. We proceed as in Section 3.

In Theorem 5.1 we report an apt decomposition of the respective Hilbert space into subspaces. In contrast to Section 3 we only require two subspaces instead of three. Next we introduce in (5.10) an operator $\tilde{T}$ as an apt sign change on the subspaces. In Theorem 5.2 we report the weak $\tilde{T}$-coercivity of the modified Steklov operator function. In Corollary 5.4 we deduce convenient properties of the spectrum in $\mathbb{C}$. In contrast to $A_{X}(\cdot)$ the operator function $\tilde{A}_{\tilde{X}}(\cdot)$ is Fredholm also at the origin $\lambda=0$. Further, we require Assumption 5.3 to guarantee the discreteness of the spectrum, whereas the less restrictive Assumption 2.4 was necessary in Section 3 . We report in a companion article [16] the existence and behavior of eigenvalues for purely real, symmetric $\mu$ and $\epsilon$, i.e. in the selfadjoint case.

In Subsection 5.1 we introduce a reformulation of the eigenvalue problem by means of an auxiliary variable. This formulation avoids the explicit appearance of the operator $S$. We prove respective properties of the eigenvalue problem and its approximation. Our results in this section are similar to those of Section 3 from [12]. However, we apply a different technique: [12] reformulates the eigenvalue problem to a problem which is solely posed on the boundary. Instead we keep the natural volumetric setting, which is of importance to conduct an approximation analysis (see Sect. 6).

The modified electromagnetic Steklov eigenvalue problem is to

$$
\text { find }(\lambda, u) \in \mathbb{C} \times H(\operatorname{curl} ; \Omega) \backslash\{0\} \quad \text { so that } \tilde{A}(\lambda) u=0,
$$

whereby $\tilde{A}(\lambda) \in L(H(\operatorname{curl} ; \Omega))$ is defined through

$$
\begin{aligned}
\left\langle\tilde{A}(\lambda) u, u^{\prime}\right\rangle_{H(\operatorname{curl} ; \Omega)}:= & \left\langle\mu^{-1} \operatorname{curl} u, \operatorname{curl} u^{\prime}\right\rangle_{\mathbf{L}^{2}(\Omega)}-\omega^{2}\left\langle\epsilon u, u^{\prime}\right\rangle_{\mathbf{L}^{2}(\Omega)} \\
& -\lambda\left\langle S u, S u^{\prime}\right\rangle_{\mathbf{L}_{t}^{2}(\partial \Omega)} \text { for all } u, u^{\prime} \in H(\operatorname{curl} ; \Omega), \lambda \in \mathbb{C}
\end{aligned}
$$

and $S$ is as defined in (2.13). We note again that the sign of $\lambda$ herein is reversed compared to [12]. Also, we employ $\operatorname{tr}_{\nu \times} u$ opposed to $u_{\nu}=\operatorname{tr}_{\nu \times} u \times \nu$ in [12] and hence we employ through $S$ a map onto gradient functions opposed to a map onto curl functions as in [12]. As in Section 3 we introduce apt subspaces of $H(\operatorname{curl} ; \Omega)$ :

$$
\begin{aligned}
\tilde{V} & :=H\left(\operatorname{curl}, \operatorname{div}^{0}, \operatorname{tr}_{\nu}^{0} ; \Omega\right), \\
\tilde{W} & :=H\left(\operatorname{curl}^{0} ; \Omega\right)=\nabla H^{1}(\Omega) .
\end{aligned}
$$

Theorem 5.1. It holds

$$
H(\operatorname{curl} ; \Omega)=\tilde{V} \oplus^{\perp_{H(\operatorname{curl} ; \Omega)}} \tilde{W}
$$

i.e. the orthogonal projection operators $P_{\tilde{V}}, P_{\tilde{W}} \in L(H(\operatorname{curl} ; \Omega))$ satisfy $\operatorname{ran} P_{\tilde{V}}=\tilde{V}, \operatorname{ran} P_{\tilde{W}}=\tilde{W}, \tilde{W}=\operatorname{ker} P_{\tilde{V}}$, $\tilde{V}=\operatorname{ker} P_{\tilde{W}}, u=P_{\tilde{V}} u+P_{\tilde{W}} u$ for each $u \in H(\operatorname{curl} ; \Omega)$ and

$$
\left\langle u, u^{\prime}\right\rangle_{\tilde{X}}:=\left\langle P_{\tilde{V}} u, P_{\tilde{V}} u^{\prime}\right\rangle_{H(\operatorname{curl} ; \Omega)}+\left\langle P_{\tilde{W}} u, P_{\tilde{W}} u^{\prime}\right\rangle_{H(\operatorname{curl} ; \Omega)}=\left\langle u, u^{\prime}\right\rangle_{H(\operatorname{curl} ; \Omega)}
$$

for all $u, u^{\prime} \in H(\operatorname{curl} ; \Omega)$.

Proof. All properties are due to the orthogonal decomposition.

We observe $\tilde{W} \subset \operatorname{ker} S$. We proceed further as in Section 3. Let

$$
\tilde{X}:=H(\operatorname{curl} ; \Omega), \quad\langle\cdot, \cdot\rangle_{\tilde{X}} \quad \text { as defined in }(5.5) .
$$


Let further $\tilde{A}_{\tilde{X}}(\cdot), \tilde{A}_{c}, \tilde{A}_{\epsilon}, \tilde{A}_{l^{2}}, \tilde{A}_{\text {tr }} \in L(\tilde{X})$ be defined through

$$
\begin{aligned}
\left\langle\tilde{A}_{\tilde{X}}(\lambda) u, u^{\prime}\right\rangle_{\tilde{X}} & :=\left\langle\tilde{A}(\lambda) u, u^{\prime}\right\rangle_{H(\operatorname{curl} ; \Omega)} & & \text { for all } u, u^{\prime} \in \tilde{X}, \lambda \in \mathbb{C}, \\
\left\langle\tilde{A}_{c} u, u^{\prime}\right\rangle_{\tilde{X}} & :=\left\langle\mu^{-1} \operatorname{curl} u, \operatorname{curl} u^{\prime}\right\rangle_{\mathbf{L}^{2}(\Omega)} & & \text { for all } u, u^{\prime} \in \tilde{X} \\
\left\langle\tilde{A}_{\epsilon} u, u^{\prime}\right\rangle_{\tilde{X}} & :=\left\langle\epsilon u, u^{\prime}\right\rangle_{\mathbf{L}^{2}(\Omega)} & & \text { for all } u, u^{\prime} \in \tilde{X} \\
\left\langle\tilde{A}_{l^{2}} u, u^{\prime}\right\rangle_{\tilde{X}} & :=\left\langle u, u^{\prime}\right\rangle_{\mathbf{L}^{2}(\Omega)} & & \text { for all } u, u^{\prime} \in \tilde{X} \\
\left\langle\tilde{A}_{\mathrm{tr}} u, u^{\prime}\right\rangle_{\tilde{X}} & :=\left\langle S u, S u^{\prime}\right\rangle_{\mathbf{L}_{t}^{2}(\partial \Omega)} & & \text { for all } u, u^{\prime} \in \tilde{X}
\end{aligned}
$$

From the definitions of $\tilde{V}, \tilde{W}$ and $\tilde{W} \subset \operatorname{ker} S$ we deduce that

$$
\begin{aligned}
\tilde{A}_{\tilde{X}}(\lambda)= & \left(P_{\tilde{V}}+P_{\tilde{W}}\right)\left(\tilde{A}_{c}-\omega^{2} \tilde{A}_{\epsilon}-\lambda \tilde{A}_{\mathrm{tr}}\right)\left(P_{\tilde{V}}+P_{\tilde{W}}\right) \\
= & \mathbf{P}_{\tilde{\mathbf{V}}} \tilde{\mathbf{A}}_{\mathbf{c}} \mathbf{P}_{\tilde{\mathbf{V}}}-\omega^{2} P_{\tilde{V}} \tilde{A}_{\epsilon} P_{\tilde{V}}-\lambda P_{\tilde{V}} \tilde{A}_{\mathrm{tr}} P_{\tilde{V}}-\omega^{2} \mathbf{P}_{\tilde{\mathbf{W}}} \tilde{\mathbf{A}}_{\epsilon} \mathbf{P}_{\tilde{\mathbf{W}}} \\
& -\omega^{2}\left(P_{\tilde{W}} \tilde{A}_{\epsilon} P_{\tilde{V}}+P_{\tilde{V}} \tilde{A}_{\epsilon} P_{\tilde{W}}\right) .
\end{aligned}
$$

If we identify $\tilde{X} \sim \tilde{V} \times \tilde{W}$ and $\tilde{X} \ni u \sim(v, w) \in \tilde{V} \times \tilde{W}$, we can identify $\tilde{A}_{\tilde{X}}(\lambda)$ with the block operator

$$
\left(\begin{array}{cc}
\left.\mathbf{P}_{\tilde{\mathbf{V}}} \tilde{\mathbf{A}}_{\mathbf{c}}\right|_{\tilde{\mathbf{V}}}-\left.P_{\tilde{V}}\left(\omega^{2} \tilde{A}_{\epsilon}+\lambda \tilde{A}_{\mathrm{tr}}\right)\right|_{\tilde{V}}-\left.\omega^{2} P_{\tilde{V}} \tilde{A}_{\epsilon}\right|_{\tilde{W}} \\
-\left.\omega^{2} P_{\tilde{W}} \tilde{A}_{\epsilon}\right|_{\tilde{V}} & -\left.\omega^{2} \mathbf{P}_{\tilde{\mathbf{W}}} \tilde{\mathbf{A}}_{\epsilon}\right|_{\tilde{\mathbf{W}}}
\end{array}\right) .
$$

We highlighted with color and boldface in (5.8) and (5.9) the operators which are not compact. This leads us to define a test function operator in the following way. Let

$$
\tilde{T}:=P_{\tilde{V}}-\omega^{-2} P_{\tilde{W}}
$$

Obviously $\tilde{T} \in L(\tilde{X})$ is bijective with $\tilde{T}^{-1}=P_{\tilde{V}}-\omega^{2} P_{\tilde{W}}$.

Theorem 5.2. Let $\epsilon$ satisfy Assumption 2.1, $\mu$ satisfy Assumption 2.2 and $\Omega$ satisfy Assumption 2.3. Thence $\tilde{A}_{\tilde{X}}(\cdot): \mathbb{C} \rightarrow L(\tilde{X})$ is weakly $\tilde{T}$-coercive.

Proof. Let $\lambda \in \mathbb{C}$. Set

$$
A_{1}:=P_{\tilde{V}} \tilde{A}_{c} P_{\tilde{V}}+P_{\tilde{V}} \tilde{A}_{l^{2}} P_{\tilde{V}}-\omega^{2} P_{\tilde{W}} \tilde{A}_{\epsilon} P_{\tilde{W}}
$$

and

$$
A_{2}:=-P_{\tilde{V}} \tilde{A}_{l^{2}} P_{\tilde{V}}-\omega^{2} P_{\tilde{V}} \tilde{A}_{\epsilon} P_{\tilde{V}}-\lambda P_{\tilde{V}} \tilde{A}_{\mathrm{tr}} P_{\tilde{V}}-\omega^{2}\left(P_{\tilde{W}} \tilde{A}_{\epsilon} P_{\tilde{V}}+P_{\tilde{V}} \tilde{A}_{\epsilon} P_{\tilde{W}}\right)
$$

so that $\tilde{A}_{\tilde{X}}(\lambda)=A_{1}+A_{2}$.

To see that $P_{\tilde{V}} \tilde{A}_{\mathrm{tr}} P_{\tilde{V}}$ is a compact operator we recall $\tilde{A}_{\mathrm{tr}}=S^{*} S$ from $(5.7 \mathrm{e})$ and $S P_{\tilde{V}}=P_{\nabla_{\partial}} \operatorname{tr}_{\nu \times} P_{\tilde{V}}$ from (2.14). It follows now by means of (2.9) that $S P_{\tilde{V}}$ is compact and hence $P_{\tilde{V}} \tilde{A}_{\mathrm{tr}} P_{\tilde{V}}$ is compact too. The remaining terms of $A_{2}$ are compact due to (2.8). Hence $T(\lambda)^{*} A_{2}$ is compact too. It is straight forward to see

$$
\Re\left(\left\langle A_{1} u, \tilde{T} u\right\rangle_{\tilde{X}}\right) \geq \min \left(1, c_{\epsilon}, c_{\mu}\right)\|u\|_{\tilde{X}}^{2}
$$

i.e. $\tilde{T}^{*} A_{1}$ is coercive.

As in [12] we impose an additional assumption.

Assumption 5.3. Let $\tilde{A}_{\tilde{X}}(0)$ be injective. 
Assumption 5.3 is equivalent to the statement that $\omega^{2}$ is not an eigenvalue to the Neumann eigenvalue problem

$$
\begin{array}{rlrl}
\operatorname{curl} \mu^{-1} \operatorname{curl} u-\omega^{2} \epsilon u & =0 & & \text { in } \Omega, \\
\nu \times \operatorname{curl} u=0 & & \text { at } \partial \Omega .
\end{array}
$$

Since it is known that the eigenvalues to this problem form only a discrete set, Assumption 5.3 is reasonable.

Corollary 5.4. Let Assumptions 2.1-2.3 and 5.3 hold true. Then $\tilde{A}_{\tilde{X}}(\lambda)$ is bijective for all $\lambda \in \mathbb{C}$ with $\Im(\lambda)<0$ and $\lambda=0$. The spectrum of $\tilde{A}_{\tilde{X}}(\cdot)$ in $\mathbb{C}$ consists of an at most countable set of eigenvalues with finite algebraic multiplicity which have no accumulation point in $\mathbb{C}$.

Proof. Let $\lambda \in \mathbb{C}$ with $\Im(\lambda)<0$ and $u \in X$ be so that $\tilde{A}_{\tilde{X}}(\lambda) u=0$. It follows

$$
\left.0=-\Im\left(\left\langle\tilde{A}_{\tilde{X}}(\lambda) u, u\right)\right\rangle_{\tilde{X}}\right) \geq-\Im(\lambda)\|S u\|_{\mathbf{L}_{t}^{2}(\partial \Omega)}^{2}
$$

and hence $\tilde{A}_{\tilde{X}}(0) u=\tilde{A}_{\tilde{X}}(\lambda) u=0$. Due to Assumption 5.3 it follows $u=0$, i.e. $\tilde{A}_{\tilde{X}}(\lambda)$ is injective. From Theorem 5.2 we know that $\tilde{A}_{\tilde{X}}(\lambda)$ is Fredholm with index zero for all $\lambda \in \mathbb{C}$ and hence $\tilde{A}_{\tilde{X}}(\lambda)$ is bijective, if $\Im(\lambda)<0$ or $\lambda=0$. For the remaining claim see the proof of Corollary 5.4.

\subsection{Auxiliary formulation}

A Galerkin approximation to (5.1) doesn't yield a computational method yet, because the term $\left\langle S u_{n}, S u_{n}^{\prime}\right\rangle_{\mathbf{L}_{t}^{2}(\partial \Omega)}$ needs to be evaluated. Therefore we proceed as in [12] and introduce an auxiliary variable. To this end let

$$
\begin{aligned}
& \tilde{Z}:=H_{*}^{1}(\partial \Omega), \quad\langle\cdot, \cdot\rangle_{\tilde{Z}}:=\left\langle\nabla_{\partial} \cdot \nabla_{\partial} \cdot\right\rangle_{\mathbf{L}_{t}^{2}(\partial \Omega)}, \\
& \tilde{X}:=\tilde{X} \times \tilde{Z}, \quad\left\langle(u, z),\left(u^{\prime}, z^{\prime}\right)\right\rangle_{\tilde{X}}:=\left\langle u, u^{\prime}\right\rangle_{\tilde{X}}+\left\langle z, z^{\prime}\right\rangle_{\tilde{Z}}
\end{aligned}
$$

for all $(u, z),\left(u^{\prime}, z^{\prime}\right) \in \tilde{X}$ and for $l \in\{0,1\}$ let

$$
\begin{aligned}
\left\langle\tilde{A}^{l}(\lambda)(u, z),\left(u^{\prime}, z^{\prime}\right)\right\rangle_{\tilde{X}}:= & \left\langle\mu^{-1} \operatorname{curl} u, \operatorname{curl} u^{\prime}\right\rangle_{\mathbf{L}^{2}(\Omega)}-\omega^{2}\left\langle\epsilon u, u^{\prime}\right\rangle_{\mathbf{L}^{2}(\Omega)} \\
& +\lambda\left\langle z, \operatorname{div}_{\partial} \operatorname{tr}_{\nu \times} u^{\prime}\right\rangle_{H^{1}(\partial \Omega) \times H^{-1}(\partial \Omega)} \\
& +\lambda^{l}\left\langle\operatorname{div}_{\partial} \operatorname{tr}_{\nu \times} u, z^{\prime}\right\rangle_{H^{-1}(\partial \Omega) \times H^{1}(\partial \Omega)} \\
& +\lambda^{l}\left\langle\nabla_{\partial} z, \nabla_{\partial} z^{\prime}\right\rangle_{\mathbf{L}_{t}^{2}(\partial \Omega)}
\end{aligned}
$$

for all $(u, z),\left(u^{\prime}, z^{\prime}\right) \in \tilde{X}$ and $\lambda \in \mathbb{C}$. If the coefficients $\mu, \epsilon$ are real and symmetric, the choice $l=1$ preserves the self adjointness of (5.13). This is of advantage, if one chooses to implement a discretization which is based directly on (5.13). On the other hand if one aims to build the Schur-complement with respect to the second component in a later discretization step, then the choice $l=0$ leads to no restriction on $\lambda$. Let

$$
\Lambda_{0}:=\mathbb{C}, \quad \Lambda_{1}:=\mathbb{C} \backslash\{0\} .
$$

Lemma 5.5. If $(\lambda, u) \in \mathbb{C} \times \tilde{X} \backslash\{0\}$ so that $\tilde{A}(\lambda) u=0$, then $\tilde{A}^{l}(\lambda)(u, z)=0$ with $z \in \tilde{Z}$ so that $S u=\nabla_{\partial} z$. Vice-versa, if $(\lambda,(u, z)) \in \Lambda_{l} \times \tilde{X} \backslash\{0\}$ so that $\tilde{\tilde{A}}^{l}(\lambda)(u, z)=0$, then $S u=\nabla_{\partial} z$ and $\tilde{A}(\lambda) u=0$.

Proof. Let $(\lambda, u) \in \mathbb{C} \times \tilde{X} \backslash\{0\}$ so that $\tilde{A}(\lambda) u=0$ and $z \in \tilde{Z}$ be so that $\nabla_{\partial} z=S u$. It follows

$$
\begin{aligned}
0 & =\left\langle\mu^{-1} \operatorname{curl} u, \operatorname{curl} u^{\prime}\right\rangle_{\mathbf{L}^{2}(\Omega)}-\omega^{2}\left\langle\epsilon u, u^{\prime}\right\rangle_{\mathbf{L}^{2}(\Omega)}-\lambda\left\langle S u, S u^{\prime}\right\rangle_{\mathbf{L}_{t}^{2}(\partial \Omega)} \\
& =\left\langle\mu^{-1} \operatorname{curl} u, \operatorname{curl} u^{\prime}\right\rangle_{\mathbf{L}^{2}(\Omega)}-\omega^{2}\left\langle\epsilon u, u^{\prime}\right\rangle_{\mathbf{L}^{2}(\Omega)}-\lambda\left\langle\nabla_{\partial} z, S u^{\prime}\right\rangle_{\mathbf{L}_{t}^{2}(\partial \Omega)} \\
& =\left\langle\mu^{-1} \operatorname{curl} u, \operatorname{curl} u^{\prime}\right\rangle_{\mathbf{L}^{2}(\Omega)}-\omega^{2}\left\langle\epsilon u, u^{\prime}\right\rangle_{\mathbf{L}^{2}(\Omega)}+\lambda\left\langle z, \operatorname{div}_{\partial} S u^{\prime}\right\rangle_{H^{1}(\partial \Omega) \times H^{-1}(\partial \Omega)} \\
& =\left\langle\mu^{-1} \operatorname{curl} u, \operatorname{curl} u^{\prime}\right\rangle_{\mathbf{L}^{2}(\Omega)}-\omega^{2}\left\langle\epsilon u, u^{\prime}\right\rangle_{\mathbf{L}^{2}(\Omega)}+\lambda\left\langle z, \operatorname{div}_{\partial} \operatorname{tr}_{\nu \times} u^{\prime}\right\rangle_{H^{1}(\partial \Omega) \times H^{-1}(\partial \Omega)}
\end{aligned}
$$


for each $u^{\prime} \in \tilde{X}$. It follows further

$$
0=\left\langle\operatorname{div}_{\partial} \operatorname{tr}_{\nu \times} u, z^{\prime}\right\rangle_{H^{-1}(\partial \Omega) \times H^{1}(\partial \Omega)}+\left\langle\nabla_{\partial} z, \nabla_{\partial} z^{\prime}\right\rangle_{\mathbf{L}_{t}^{2}(\partial \Omega)}
$$

for each $z^{\prime} \in \tilde{Z}$ from the definition of $S$ and $z$. The reverse direction follows like-wise.

Let $B \in L(\tilde{Z}, \tilde{X})$ so that

$$
\langle B z, u\rangle_{\tilde{X}}:=\left\langle z, \operatorname{div}_{\partial} \operatorname{tr}_{\nu \times} u\right\rangle_{H^{1}(\partial \Omega) \times H^{-1}(\partial \Omega)}
$$

for all $z \in \tilde{Z}, u \in \tilde{X}$. Then $\tilde{A}^{l}(\lambda)$ admits the block representation

$$
\tilde{A}^{l}(\lambda)=\left(\begin{array}{cc}
\tilde{A}_{c}-\omega^{2} \tilde{A}_{\epsilon} & \lambda B \\
\lambda^{l} B^{*} & \lambda^{l} \mathrm{I}_{\tilde{Z}}
\end{array}\right)
$$

This leads us to define

$$
\tilde{\tilde{T}}^{l}(\lambda):=\left(\tilde{T}_{\bar{\lambda}^{-l} \mathrm{I}_{\tilde{Z}}}\right), \quad \lambda \in \Lambda_{l}
$$

Theorem 5.6. Let $\epsilon$ satisfy Assumption 2.1, $\mu$ satisfy Assumption 2.2 and $\Omega$ satisfy Assumption 2.3. Thence $\tilde{\tilde{A}}^{l}(\cdot): \Lambda_{l} \rightarrow L(\tilde{X})$ is weakly $\tilde{\tilde{T}}^{l}(\cdot)$-coercive.

Proof. Let

$$
A_{1}:=\left(P_{\tilde{V}}\left(\tilde{A}_{c}+\tilde{A}_{l^{2}}\right) P_{\tilde{V}}-\omega^{2} P_{\tilde{W}} \tilde{A}_{\epsilon} P_{\tilde{W}} \lambda^{l} \mathrm{I}_{\tilde{Z}}\right)
$$

and

$$
A_{2}:=\left(-P_{\tilde{V}}\left(\omega^{2} \tilde{A}_{\epsilon}+\tilde{A}_{l^{2}}\right) P_{\tilde{V}}-\omega^{2}\left(P_{\tilde{V}} \tilde{A}_{\epsilon} P_{\tilde{W}}+P_{\tilde{W}} \tilde{A}_{\epsilon} P_{\tilde{V}}\right) \lambda B \lambda^{l} B^{*}\right)
$$

so that $\tilde{A}^{l}(\lambda)=A_{1}+A_{2}$. It follows

$$
\Re\left(\left\langle A_{1}(u, z), \tilde{T}^{l}(\lambda)(u, z)\right\rangle_{\tilde{X}}\right) \geq \min \left(1, c_{\mu}, c_{\epsilon}\right)\|(u, z)\|_{\tilde{X}}^{2}
$$

for each $(u, z) \in \tilde{\tilde{X}}$, i.e. $\tilde{\tilde{T}}^{l}(\lambda)^{*} A_{1}$ is coercive. Let $\iota \in L\left(H^{-1 / 2}(\partial \Omega), H^{1 / 2}(\partial \Omega)\right)$ be the isomorphism so that $\left\langle\phi, \phi^{\prime}\right\rangle_{H^{1 / 2}(\partial \Omega) \times H^{-1 / 2}(\partial \Omega)}=\left\langle\phi, \iota \phi^{\prime}\right\rangle_{H^{1 / 2}(\partial \Omega)}$ for all $\phi \in H^{1 / 2}(\partial \Omega)$ and $\phi^{\prime} \in H^{-1 / 2}(\partial \Omega)$. Let $E \in$ $L\left(H^{1}(\partial \Omega), H^{1 / 2}(\partial \Omega)\right)$ be the embedding operator. Then

$$
\begin{aligned}
\langle B z, u\rangle_{\tilde{X}} & =\left\langle z, \operatorname{div}_{\partial} \operatorname{tr}_{\nu \times} u\right\rangle_{H^{1}(\partial \Omega) \times H^{-1}(\partial \Omega)} \\
& =\left\langle E z, \operatorname{div}_{\partial} \operatorname{tr}_{\nu \times} u\right\rangle_{H^{1 / 2}(\partial \Omega) \times H^{-1 / 2}(\partial \Omega)} \\
& =\left\langle E z, \iota \operatorname{div}_{\partial} \operatorname{tr}_{\nu \times} u\right\rangle_{H^{1 / 2}(\partial \Omega)} \\
& =\left\langle\left(\iota \operatorname{div}_{\partial} \operatorname{tr}_{\nu \times}\right)^{*} E z, u\right\rangle_{\tilde{X}},
\end{aligned}
$$

i.e. $B=\left(\iota \operatorname{div}_{\partial} \operatorname{tr}_{\nu \times}\right)^{*} E$. Since $E$ is compact, so are $B$ and $B^{*}$. The remaining terms of $A_{2}$ are compact due to (2.8). Hence $\tilde{\tilde{T}}^{l}(\lambda)^{*} A_{2}$ is compact too.

Corollary 5.7. Let Assumptions 2.1-2.3 and 5.3 hold true. Then $\tilde{\tilde{A}}^{l}(\lambda)$ is bijective for all $\lambda \in \mathbb{C}$ with $\Im(\lambda)<0$.

Proof. Follows from Theorem 5.6, Lemma 5.5 and Corollary 5.4. 


\section{Compatible approximation of the modified Steklov eigenvalue problem}

In this section we discuss Galerkin approximations of $\tilde{\tilde{A}}^{l}(\cdot)$. We proceed as in Section 4 . We embrace the basic Assumptions 6.1, 6.2 and in Assumption 6.3 the existence of uniformly bounded commuting projections like in [2]. We report in Corollary 6.6 that for Galerkin approximations which satisfy these three assumptions, we can construct a sequence of operator functions $\tilde{T}_{n}^{l}(\lambda) \in L\left(\tilde{X}_{n}\right)$ which converges to $\tilde{T}^{l}(\lambda)$ in discrete norm $(2.1)$ at each $\lambda \in \Lambda_{l}$. Consequently we report in Theorem 6.7 that the abstract framework of [17] is applicable. Finally, we discuss some topics concerning the computational implementation.

The investigated computational method in this section is the one proposed in [12]. We amend the results of [12] with a convergence analysis of their method.

Consider the following basic assumptions.

Assumption 6.1. Let $\left(\tilde{X}_{n}\right)_{n \in \mathbb{N}}$ be so that $\tilde{X}_{n} \subset \tilde{X}$, $\operatorname{dim} \tilde{X}_{n}<\infty$ for each $n \in \mathbb{N}$ and

$$
\lim _{n \in \mathbb{N}} \inf _{u^{\prime} \in \tilde{X}_{n}}\left\|u-u^{\prime}\right\|_{\tilde{X}}=0 \text { for each } u \in \tilde{X} .
$$

Assumption 6.2. Let $\left(\tilde{Z}_{n}\right)_{n \in \mathbb{N}}$ be so that $\tilde{Z}_{n} \subset \tilde{Z}$, $\operatorname{dim} \tilde{Z}_{n}<\infty$ for each $n \in \mathbb{N}$ and

$$
\lim _{n \in \mathbb{N}} \inf _{z^{\prime} \in \tilde{Z}_{n}}\left\|z-z^{\prime}\right\|_{\tilde{Z}}=0 \text { for each } z \in \tilde{Z} .
$$

Let

$$
\tilde{\tilde{X}}_{n}:=\tilde{X}_{n} \times \tilde{Z}_{n}
$$

Consider the following additional assumption.

Assumption 6.3. There exists $\left(\pi_{n}^{\tilde{X}}\right)_{n \in \mathbb{N}}$ so that

$$
\begin{aligned}
& \pi_{n}^{\tilde{X}} \in L\left(\mathbf{L}^{2}(\Omega)\right) \text { is a projector with } \tilde{X}_{n}=\operatorname{ran} \pi_{n}^{\tilde{X}}, \\
& \sup _{n \in \mathbb{N}}\left\|\pi_{n}^{\tilde{X}}\right\|_{L\left(\mathbf{L}^{2}(\Omega)\right)}<+\infty .
\end{aligned}
$$

Let $\tilde{Y}:=\mathbf{L}^{2}(\Omega)$. There exist sequences $\left(\tilde{Y}_{n}, \pi_{n}^{\tilde{Y}}\right)_{n \in \mathbb{N}}$ so that

$$
\begin{array}{r}
\tilde{Y}_{n} \subset Y, \quad \lim _{n \in \mathbb{N}} \inf _{u^{\prime} \in \tilde{Y}_{n}}\left\|u-u^{\prime}\right\|_{\tilde{Y}}=0, \\
\pi_{n}^{\tilde{Y}} \in L(\tilde{Y}) \text { is a projector with } \tilde{Y}_{n} \subset \operatorname{ran} \pi_{n}^{\tilde{Y}}, \\
\sup _{n \in \mathbb{N}}\left\|\pi_{n}^{\tilde{Y}}\right\|_{L(\tilde{Y})}<+\infty .
\end{array}
$$

Denote $\tilde{E} \in L\left(\tilde{X}, \mathbf{L}^{2}(\Omega)\right)$ the embedding operator and set

$$
\tilde{\pi}_{n}:=\pi_{n}^{\tilde{X}} \tilde{E} .
$$

Further let

$$
\operatorname{curl} \circ \tilde{\pi}_{n} u=\pi_{n}^{\tilde{Y}} \circ \operatorname{curl} u
$$

for each $u \in \tilde{X}$.

Lemma 6.4. Let Assumptions 6.1 and 6.3 hold true. Then the projections $\pi_{n}^{\tilde{X}}$ and $\pi_{n}^{\tilde{Y}}$ converge point-wise to the identity in $\mathbf{L}^{2}(\Omega)$.

Proof. Proceed as for Lemma 4.3. 
Lemma 6.5. Let Assumptions 2.1, 2.3, 6.1 and 6.3 hold true. Then

$$
\begin{aligned}
& \lim _{n \in \mathbb{N}} \inf _{u \in \tilde{X}_{n} \backslash\{0\}}\left\|\left(1-\tilde{\pi}_{n}\right) P_{\tilde{V}} u\right\|_{\tilde{X}} /\|u\|_{\tilde{X}}=0, \\
& \lim _{n \in \mathbb{N}} \inf _{u \in \tilde{X}_{n} \backslash\{0\}}\left\|\left(1-\tilde{\pi}_{n}\right) P_{\tilde{W}} u\right\|_{\tilde{X}} /\|u\|_{\tilde{X}}=0 .
\end{aligned}
$$

Proof. Proceed as for Lemma 4.4.

Corollary 6.6. Let Assumptions 2.1, 2.3, 6.1, 6.2 and 6.3 hold true. Let $\tilde{T}_{n} \in L\left(\tilde{X}_{n}\right)$ be defined as $\tilde{T}_{n}:=$ $\left.\tilde{\pi}_{n} \tilde{T}\right|_{\tilde{X}_{n}}$ and $\tilde{T}_{n}^{l}(\lambda) \in L\left(\tilde{X}_{n}\right)$ as

$$
\tilde{\tilde{T}}_{n}^{l}(\lambda):=\left(\begin{array}{lll}
\tilde{T}_{n} & & \\
& \bar{\lambda}^{-l} & \\
& & \mathrm{I}_{\tilde{Z}_{n}}
\end{array}\right) .
$$

for $\lambda \in \Lambda_{l}$. Then

$$
\lim _{n \in \mathbb{N}}\left\|\tilde{T}^{l}(\lambda)-\tilde{T}_{n}^{l}(\lambda)\right\|_{n}=0
$$

at each $\lambda \in \Lambda_{l}$.

Proof. Proceed as for Corollary 4.5.

Theorem 6.7. Let Assumptions 2.1-2.3 and 5.3 hold true. Let $\tilde{X}, \tilde{A}^{l}(\cdot), \tilde{T}^{l}(\cdot)$ and $\Lambda_{l}$ be as defined in (5.11), (5.13), (5.17) and (5.14) respectively. Let Assumptions 6.1-6.3 hold true. Then $\tilde{\tilde{A}}^{l}(\cdot): \Lambda_{l} \rightarrow L(\tilde{X})$ is a holomorphic weakly $\tilde{T}^{l}(\cdot)$-coercive operator function with non-empty resolvent set and the sequence of Galerkin approximations $\left(\left.\tilde{\tilde{P}}_{n} \tilde{\widetilde{A}}^{l}(\cdot)\right|_{\tilde{X}_{n}}: \Lambda_{l} \rightarrow L\left(\tilde{X}_{n}\right)\right)_{n \in \mathbb{N}}$ is $\tilde{\tilde{T}}^{l}(\cdot)$-compatible.

Thus Corollary 2.8 of [1 $1^{n}$ ] is applicable and the convergence statements (i)-(vii) formulated in Subsection 2.1 hold.

Proof. Follows from Theorem 5.6, Corollaries 5.7 and 6.6.

The consequence of Theorem 6.7 is that if we approximate Eigenvalue Problem (5.1) by means of common $H$ (curl)-finite element spaces [2], then Assumption 6.3 is satisfied and we obtain asymptotically reliable approximations. Again, we emphasize that the commuting projection operators are only a theoretical tool to conduct the analysis and are not required for the actual implementation of the finite element method. Thus, different than for the original eigenvalue problem our results for the modified eigenvalue problem are quite satisfactory. In particular we can answer a specific question from [12]: If $\tilde{X}_{n}$ and $\tilde{Z}_{n}$ are chosen as finite element spaces with fixed polynomial degrees $p_{\tilde{X}}, p_{\tilde{Z}}$ and decreasing mesh width $h(n)$, Theorem 6.7 tells that one should choose $p_{\tilde{X}}=p_{\tilde{Z}}$ to obtain asymptotically optimal convergence rates.

We move on and discuss further issues related to the computational implementation. We note that if $\tilde{X}_{n} \subset$ $H\left(\right.$ curl, $\left.\operatorname{tr}_{\nu \times} ; \Omega\right)$, then the duality pairs in (5.13) can be evaluated as integrals:

$$
\left\langle z_{n}, \operatorname{div}_{\partial} \operatorname{tr}_{\nu \times} u_{n}\right\rangle_{H^{1}(\partial \Omega) \times H^{-1}(\partial \Omega)}=-\left\langle\nabla_{\partial} z_{n}, \operatorname{tr}_{\nu \times} u_{n}\right\rangle_{\mathbf{L}_{t}^{2}(\partial \Omega)}
$$

Let further for $u_{n} \in \tilde{X}_{n}, z_{n}$ be the solution to find $z_{n} \in \tilde{Z}_{n}$ so that

$$
\left\langle\nabla_{\partial} z_{n}, \nabla_{\partial} z_{n}^{\prime}\right\rangle_{\mathbf{L}_{t}^{2}(\partial \Omega)}=-\left\langle\operatorname{div}_{\partial} \operatorname{tr}_{\nu \times} u_{n}, z_{n}^{\prime}\right\rangle_{H^{-1}(\partial \Omega) \times H^{1}(\partial \Omega)}
$$

for all $z_{n}^{\prime} \in \tilde{Z}_{n}$ and set

$$
S_{n} u:=\nabla_{\partial} z_{n} .
$$

From the construction of $S_{n}$ it follows $S_{n} \in L\left(\tilde{X}_{n}, \mathbf{L}_{t}^{2}(\partial \Omega)\right)$ and further

$$
S_{n} u=P_{\nabla_{\partial}}^{n} \operatorname{tr}_{\nu \times} u_{n}
$$


for $u_{n} \in H\left(\operatorname{curl}, \operatorname{tr}_{\nu \times} ; \Omega\right)$ with $P_{\nabla_{\partial}}^{n}$ being the $\mathbf{L}_{t}^{2}(\partial \Omega)$-orthogonal projection onto $\nabla \tilde{Z}_{n}$. Let further $\tilde{A}_{n}(\lambda) \in$ $L\left(\tilde{X}_{n}\right)$ be defined by

$$
\begin{aligned}
\left\langle\tilde{A}_{n}(\lambda) u_{n}, u_{n}^{\prime}\right\rangle_{\tilde{X}}:= & \left\langle\mu^{-1} \operatorname{curl} u_{n}, \operatorname{curl} u_{n}^{\prime}\right\rangle_{\mathbf{L}_{t}^{2}(\partial \Omega)}-\omega^{2}\left\langle\epsilon u_{n}, u_{n}^{\prime}\right\rangle_{\mathbf{L}_{t}^{2}(\partial \Omega)} \\
& -\lambda\left\langle S_{n} u_{n}, S_{n} u_{n}^{\prime}\right\rangle_{\mathbf{L}_{t}^{2}(\partial \Omega)} \text { for all } u_{n}, u_{n}^{\prime} \in \tilde{X}_{n}, \lambda \in \mathbb{C},
\end{aligned}
$$

i.e. $\tilde{A}_{n}(\lambda)$ is the Schur-complement of $\left.\tilde{\tilde{P}}_{n} \tilde{\tilde{A}}^{0}(\lambda)\right|_{\tilde{X}_{n}}$ with respect to $z_{n} \in \tilde{Z}_{n}$. Obviously $\tilde{A}_{n}(\cdot)$ is a Galerkin approximation with variational crime $S_{n}^{*} S_{n} \neq\left.\tilde{P}_{n} S^{*} S\right|_{\tilde{X}_{n}}$ of $\tilde{A}(\cdot)$. The approximation properties of $\tilde{A}_{n}(\cdot)$ to $\tilde{A}(\cdot)$ are already provided by our previous analysis, i.e. our analysis technique avoided to discuss the variational crime directly. If further $\tilde{X}_{n} \subset H\left(\operatorname{curl}, \operatorname{tr}_{\nu \times} ; \Omega\right)$, then

$$
\begin{aligned}
\left\langle S_{n} u_{n}, S_{n} u_{n}^{\prime}\right\rangle_{\mathbf{L}_{t}^{2}(\partial \Omega)} & =\left\langle P_{\nabla_{\partial}}^{n} \operatorname{tr}_{\nu \times} u_{n}, P_{\nabla_{\partial}}^{n} \operatorname{tr}_{\nu \times} u_{n}^{\prime}\right\rangle_{\mathbf{L}_{t}^{2}(\partial \Omega)} \\
& =\left\langle P_{\nabla_{\partial}}^{n} \operatorname{tr}_{\nu \times} u_{n}, \operatorname{tr}_{\nu \times} u_{n}^{\prime}\right\rangle_{\mathbf{L}_{t}^{2}(\partial \Omega)} \\
& =\left\langle S_{n} u_{n}, \operatorname{tr}_{\nu \times} u_{n}^{\prime}\right\rangle_{\mathbf{L}_{t}^{2}(\partial \Omega)} .
\end{aligned}
$$

Let $\left(z_{n}\right)_{n=1}^{N}$ be a basis of $\tilde{Z}_{n}$ and consider the matrix $M \in \mathbb{C}^{N \times N}$ with entries

$$
M_{n, m}:=\left\langle\nabla_{\partial} z_{n}, \nabla_{\partial} z_{m}\right\rangle_{\mathbf{L}_{t}^{2}(\partial \Omega)} .
$$

To implement the operator $S_{n}$, the matrix $M$ needs to be inverted. However, due to $\tilde{Z}_{n} \subset \tilde{Z}=H_{*}^{1}(\partial \Omega)$ the matrix $M$ is dense. To obtain a sparse matrix $M$ the following procedure was suggested in [12]. Let $\gamma>0$ be small and $\mathbb{K}:=\operatorname{span}\{1\}$ be the space of constant functions. For $u_{n} \in \tilde{X}_{n}$ let $z_{n}$ be the solution to find $z_{n} \in \tilde{Z}_{n} \oplus \mathbb{K} \subset H^{1}(\partial \Omega)$ so that

$$
\left\langle\nabla_{\partial} z_{n}, \nabla_{\partial} z_{n}^{\prime}\right\rangle_{\mathbf{L}_{t}^{2}(\partial \Omega)}+\gamma\left\langle z_{n}, z_{n}^{\prime}\right\rangle_{L^{2}(\partial \Omega)}=-\left\langle\operatorname{div}_{\partial} \operatorname{tr}_{\nu \times} u_{n}, z_{n}^{\prime}\right\rangle_{H^{-1}(\partial \Omega) \times H^{1}(\partial \Omega)}
$$

for all $z_{n}^{\prime} \in \tilde{Z}_{n} \oplus \mathbb{K} \subset H^{1}(\partial \Omega)$ and set

$$
S_{n}^{\gamma} u_{n}:=\nabla_{\partial} z_{n} .
$$

We analyze this modification in two steps. First we consider the perturbation of the sesquilinear form $\left\langle\nabla_{\partial}, \nabla_{\partial} \cdot\right\rangle_{\mathbf{L}_{t}^{2}(\partial \Omega)}$ to $\left\langle\nabla_{\partial} \cdot \nabla_{\partial} \cdot\right\rangle_{\mathbf{L}_{t}^{2}(\partial \Omega)}+\gamma\langle\cdot, \cdot\rangle_{L^{2}(\partial \Omega)}$ on the space $\tilde{Z}_{n} \subset H_{*}^{1}(\partial \Omega)$. The analysis of such a perturbation is straight forward and of magnitude $\gamma$. Secondly we note that the solution $z_{n} \in \tilde{Z}_{n} \oplus \mathbb{K} \subset H^{1}(\partial \Omega)$ to $(6.17)$ satisfies $\left\langle z_{n}, 1\right\rangle_{L^{2}(\partial \Omega)}=0$, i.e. $z_{n} \in \tilde{Z}_{n}$. Thus a replacement of $\tilde{Z}_{n} \subset H_{*}^{1}(\partial \Omega)$ by $\tilde{Z}_{n} \oplus \mathbb{K} \subset H^{1}(\partial \Omega)$ doesn't change the respective solution to (6.17) and hence no additional error is produced.

\section{Conclusion}

The application of electromagnetic Steklov eigenvalues intended to serve as target signatures for nondestructive testing of materials was first considered in [12]. In [16] the relation between the modified and the original electromagnetic Steklov eigenvalue problems and properties of the eigenvalue distributions were reported for the selfadjoint cases.

In this article we conducted a rigorous approximation analysis for the modified version. For the original version, we established the discreteness of the eigenvalues. We reported an abstract approximation analysis, which requires the approximation spaces to satisfy Assumption 4.2. It remains an open question if convenient $H$ (curl)-finite element spaces satisfy this property. Much more numerical testing on realistic geometries is needed to see if (modified) Steklov eigenvalues can prevail as target signature for realistic applications.

This article also examplifies how the general framework [17] can be applied to analyze approximations of linear eigenvalue problems without the introduction of a solution operator (which is the classical approach [3], $[5])$. 


\section{REFERENCES}

[1] C. Amrouche, C. Bernardi, M. Dauge and V. Girault, Vector potentials in three-dimensional non-smooth domains. Math. Methods Appl. Sci. 21 (1998) 823-864.

[2] D.N. Arnold, R.S. Falk and R. Winther, Finite element exterior calculus: from Hodge theory to numerical stability. Bull. Amer. Math. Soc. (N.S.) 47 (2010) 281-354.

[3] I. Babuška and J. Osborn, Eigenvalue problems. In: Vol. 2 of Finite Element Methods (Part 1), Handbook of Numerical Analysis. Elsevier (1991) 641-787.

[4] J.M. Ball, Y. Capdeboscq and B. Tsering-Xiao, On uniqueness for time harmonic anisotropic Maxwell's equations with piecewise regular coefficients. Math. Models Methods Appl. Sci. 22 (2012) 1250036.

[5] D. Boffi, Finite element approximation of eigenvalue problems. Acta Numer. 19 (2010) 1-120.

[6] A.-S. Bonnet-BenDhia, P. Ciarlet and C.M. Zwölf, Time harmonic wave diffraction problems in materials with sign-shifting coefficients. J. Comput. Appl. Math. 234 (2010) 1912-1919.

[7] A. Buffa, Remarks on the discretization of some noncoercive operator with applications to heterogeneous maxwell equations. SIAM J. Numer. Anal. 43 (2005) 1-18.

[8] A. Buffa, M. Costabel and D. Sheen, On traces for H(curl, $\Omega$ ) in Lipschitz domains. J. Math. Anal. Appl. 276 (2002) 845-867.

[9] F. Cakoni and D. Colton, Qualitative methods in inverse scattering theory. In: An introduction: Interaction of Mechanics and Mathematics. Springer-Verlag, Berlin-Heidelberg (2006).

[10] F. Cakoni, D. Colton, S. Meng and P. Monk, Stekloff eigenvalues in inverse scattering. SIAM J. Appl. Math. 76 (2016) $1737-1763$.

[11] F. Cakoni, D. Colton and H. Haddar, Inverse scattering theory and transmission eigenvalues. In: Vol. 88 of $C B M S-N S F$ Regional Conference Series in Applied Mathematics. Society for Industrial and Applied Mathematics (SIAM), Philadelphia, PA (2016).

[12] J. Camaño, C. Lackner and P. Monk, Electromagnetic Stekloff eigenvalues in inverse scattering. SIAM J. Math. Anal. 49 (2017) 4376-4401.

[13] M. Costabel, A remark on the regularity of solutions of Maxwell's equations on Lipschitz domains. Math. Methods Appl. Sci. 12 (1990) 365-368.

[14] M. Dauge, Elliptic boundary value problems on corner domains. In: Vol. 1341 of Smoothness and Asymptotics of Solutions. Lecture Notes in Mathematics. Springer, Berlin-Heidelberg (1988).

[15] A. Ern and J.-L. Guermond, Mollification in strongly Lipschitz domains with application to continuous and discrete de Rham complexes. Comput. Methods Appl. Math. 16 (2016) 51-75.

[16] M. Halla, Electromagnetic Stekloff eigenvalues: existence and behavior in the selfadjoint case. Preprint arXiv:1909.01983 (2019).

[17] M. Halla, Galerkin approximation of holomorphic eigenvalue problems: weak T-coercivity and T-compatibility. Preprint arXiv: $1908.05029(2019)$.

[18] O. Karma, Approximation in eigenvalue problems for holomorphic Fredholm operator functions. I. Numer. Funct. Anal. Optim. 17 (1996) 365-387.

[19] O. Karma, Approximation in eigenvalue problems for holomorphic Fredholm operator functions. II. (Convergence rate). Numer. Funct. Anal. Optim. 17 (1996) 389-408.

[20] V. Kozlov and V. Maz'ya, Differential equations with operator coefficients with applications to boundary value problems for partial differential equations. In: Springer Monographs in Mathematics. Springer, Berlin-Heidelberg (1999). 